\title{
Computer-Assisted Discovery and Proof
}

\author{
David H. Bailey and Jonathan M. Borwein
}

\begin{abstract}
With the advent of powerful, widely-available mathematical software, combined with ever-faster computer hardware, we are approaching a day when both the discovery and proof of mathematical facts can be done in a computer-assisted manner. This article presents several specific examples of this new paradigm in action.
\end{abstract}

Kronecker said "In mathematics, I recognize true scientific value only in concrete mathematical truths, or to put it more pointedly, only in mathematical formulas." I would rather say "computations" than "formulas," but my view is essentially the same. Harold Edwards, Essays in Constructive Mathematics, 2004

All truths are easy to understand once they are discovered; the point is to discover them. - attributed to Galileo Galilei

\section{Introduction}

Both of the present authors recall the time when computation was regarded as either external or irrelevant to research mathematics: "real mathematicians don't compute." But the past 10-15 years has seen an explosion of activity in what is now termed "experimental" mathematics or "computer-assisted" mathematics (henceforth we will use these terms interchangeably). At least one full-fledged journal, Experimental Mathematics, is in operation in this specific field, and publications such as Mathematics of Computation have been publishing related articles for many years. One could argue that the most persuasive indication that computer-assisted mathematics has reached maturity is that it is not unusual to see computations mentioned in almost any mathematical journal, even in very "pure" venues that have no mission to feature computational studies.

Certainly one key driving force in this phenomenon is the development and proliferation of high-quality commercial software packages such as Wolfram Research's Mathematica, Maplesoft's Maple, and MathWork's MATLAB. These firms

David H. Bailey wishes to thank the support of the Director, Office of Computational and Technology Research, Division of Mathematical, Information, and Computational Sciences of the U.S. Department of Energy, under contract number DE-AC02-05CH11231.

Jonathan M. Borwein wishes to thank the support of the NSERC and the Canada Research Chair Programme. 
continue to upgrade their products with useful packages, such as Maple's 'gfun' (a marvelous tool for manipulating and discovering generating functions), and valuable online resources, such as Mathematica's MathWorld. Numerous other mathematical software facilities are available, often freely on the Internet, including for instance the Magma algebraic computing package and the recently announced GraPHedron package for graph theory operations. A listing of many of these resources is available on the authors' experimental mathematics website, http://www . experimentalmath.info.

The other driving force behind the rise of experimental and computer-assisted mathematics is, of course, the continuing exponential increase in computer processing power. Moore's Law, that remarkable phenomenon wherein the density (and hence processing power and memory capacity) of semiconductor devices doubles roughly every 18 to 24 months, recently celebrated the 40 th anniversary of its original statement by Gordon Moore [37], and no end is yet in sight. We noted in in 2004 [19, pg. 4], that this 40-year period of sustained exponential advance has no peer in the history of technology. Since then, two additional cycles of Moore's Law have roughly quadrupled processing power and memory capacity from 2004 levels.

At the present time the computer industry in general (as well as the mathematical computing world in particular) faces a major challenge of learning to deal with parallel processing, since the foreseeable trend is for microprocessors to feature multiple "cores" (processing units) on a single chip [29]. But researchers can take comfort that the high-performance scientific computing world has been successfully dealing with parallelism for some time, as attested by the latest mind-boggling achievements noted in the Top500 list (see http://www.top500.org) and the Gordon Bell Prize (see http://awards.acm.org/bell). For example, recently an international team of researchers performed a first-principles molecular dynamics computation at 207.3 Tflop/s (i.e., 207.3 trillion floating-point operations per second), using a 131,072-CPU IBM BlueGene/L computer [33]. Large-scale parallelism has also been employed is some recent mathematical computing studies. Two such studies by the present authors are [8] and [10]. But it is fair to say that the development of efficient and user-friendly algorithmic tools for highly parallel environments is running far behind the development of the hardware.

\section{Algorithms for Experimentation}

The principal algorithms used in experimental and computer-assisted mathematics are the following:

(1) Symbolic computation for algebraic and calculus manipulations.

(2) High-precision integer and floating-point arithmetic.

(3) High-precision evaluation of integrals and infinite series summation.

(4) Integer-relation detection methods, notably the "PSLQ" algorithm.

(5) The Wilf-Zeilberger algorithm for proving summation identities.

(6) Iterative approximations to continuous functions.

(7) Identification of functions based on graph characteristics.

(8) Graphics and visualization methods targeted to mathematical objects.

We will not comment here on most of these techniques, other than to say that, as detailed in $[17, \mathbf{1 9}, \mathbf{2 0}, \mathbf{6}, \mathbf{1 8}]$ and elsewhere, all are useful in this arena, and numerous instances of them have been incorporated into commercial symbolic computing packages such as Mathematica and Maple. 
High-precision arithmetic is arguably the most heavily used single technique in this field. The main driver for this is the fact that integer detection methods (see Section 2.1), which are used to discover new mathematical identities, require very high precision numerical inputs to obtain numerically meaningful results. Many problems require several hundred digits of precision; one application required 50,000-digit precision. Computer implementations of high-precision arithmetic typically represent a high-precision datum as a string of computer words, where the first few words give the sign, exponent and array size, and the remaining words contain successive sections of the binary mantissa. A brief survey of techniques used to perform arithmetic operations on these data structures is given in $[\mathbf{1 9}, \mathrm{pg}$ 218-229].

2.1. Integer relation detection. As mentioned above, the principal driver for the need for very high-precision arithmetic-other than the sheer challenge-is the pervasive usage of integer relation computations to find underlying mathematical identities and relationships. In particular, given an $n$-long vector of real numbers $\left(x_{i}\right)$, which is typically presented as a vector of very-high-precision numerical values, an integer relation algorithm attempts to find $n$ integers $\left(a_{i}\right)$ (if a nontrivial set exists) such that

$$
a_{1} x_{1}+a_{2} x_{2}+\cdots+a_{n} x_{n}=0 .
$$

At the present time, the best-known integer relation algorithm is the "PSLQ" algorithm of Helaman Ferguson. PSLQ was named one of ten "algorithms of the century" by Computing in Science and Engineering [3]. Although the discovery of PSLQ certainly ranks as a signal accomplishment, Ferguson at the present time is even better known for his numerous mathematical-theme sculptures [40]. He sees a clear parallel between PSLQ and his sculptures-both are "subtractive" processes that reveal an underlying entity. A detailed statement of the PSLQ algorithm, together with some recently discovered "multi-level" and "multi-pair" variants of PSLQ, which run significantly faster and are better suited for parallel processing, are described in [11] and [19, pg. 230-234].

One feature of the PSLQ algorithm is that even if it fails to find a solution, it can establish that there is no relation $\left(a_{i}\right)$ with Euclidean norm less than a certain specified bound. This is often useful information by itself. It certainly warns one to avoid hunting for chimeras.

In normal operation of PSLQ on an input vector, the entries of the reduced $x$ vector gradually decrease in size until at the point of detection, the smallest entry abruptly drops to the "epsilon" of the multi-precision arithmetic being used. This behavior is shown in Figure 1, where the size drops by 180 orders of magnitude when the underlying relation is discovered. The size of this drop can be taken as a "confidence ratio" in the detection-confidence ratios of $10^{20}$ or more almost always indicate a genuine underlying mathematical relationship rather than an artifact of numerical error.

High-precision arithmetic is required for any nontrivial usage of PSLQ (or any other integer relation detection algorithm). This is because it can be readily seen that if the $n$-long input $x$ vector is known to $p$-digit precision, then almost certainly there exist random $d$-digit vectors of integers $a_{i}$ such that $a_{1} x_{1}+a_{2} x_{2}+\cdots+a_{n} x_{n}=$ 0 (to within an "epsilon" of $10^{-p}$ ), unless $p>n d$. In other words, unless the input vector $x$ is specified to greater than $d n$-digit precision, and unless arithmetic 


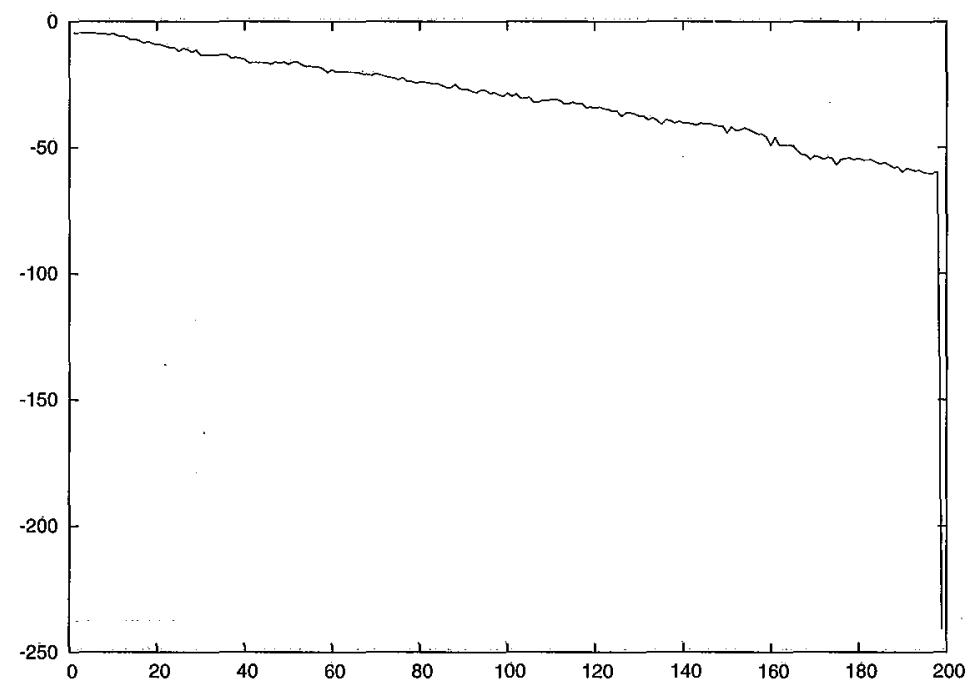

FIgURE 1. Plot of $\log _{10} \min \left|x_{i}\right|$ as a function of iteration number

operations required for the integer relation detection computation are performed to greater than $d n$-digit precision, the true underlying $n$-long, $d$-digit relation that one is seeking (if it exists) will be lost in a sea of spurious numerical artifacts.

Perhaps the best-known application of PSLQ to date is the discovery in 1996 of the "BBP" formula for $\pi$ :

$$
\pi=\sum_{n=0}^{\infty} \frac{1}{16^{n}}\left(\frac{4}{8 k+1}-\frac{2}{8 k+4}-\frac{1}{8 k+5}-\frac{1}{8 k+6}\right) .
$$

This remarkable formula permits one to calculate binary or hexadecimal digits of $\pi$ beginning at an arbitrary starting position [19, pg. 118-125]. Numerous other "BBP-type" formulas that also possess this arbitrary digit-calculating property have subsequently been discovered in a similar manner for constants such as $\zeta(2)$ (both binary and ternary), $\zeta(3)$ and Catalan's constant [4]. Many other experimental math applications of PSLQ are listed in [19, pg. 50-63] and elsewhere throughout $[\mathbf{2 0}, \mathbf{6}]$, and we will present some additional examples in this paper.

2.2. Numerical Quadrature. One other computational technique that we will briefly mention here is quadrature (i.e., numerical integration) algorithms. While a number of quadrature techniques, including such venerable methods as Gaussian quadrature, have been applied by us and others in experimental mathematics, we have found that the little-known "tanh-sinh" scheme of Mori and Takahashi possesses several notable advantages. These include the ability to produce high-precision numerical values even for integrand functions with singularities and/or infinite derivatives at endpoints, and the fact that the cost of computing the requisite abscissas and weights scales only linearly with the number of evaluation points. For many integrand functions of interest, the tanh-sinh scheme exhibits "quadratic convergence"-doubling the number of evaluation points roughly doubles the number of correct digits in quadrature results. 
The tanh-sinh quadrature scheme transforms the integral of a given function $f(x)$ on the interval $[-1,1]$ to an integral on $(-\infty, \infty)$ using the change of variable $x=g(t)$, where $g(t)=\tanh (\pi / 2 \cdot \sinh t)$. Note that $g(x)$ has the property that $g(x) \rightarrow 1$ as $x \rightarrow \infty$ and $g(x) \rightarrow-1$ as $x \rightarrow-\infty$, and all derivatives rapidly approach zero for large positive and negative arguments. Thus one can write, for $h>0$,

$$
\int_{-1}^{1} f(x) d x=\int_{-\infty}^{\infty} f(g(t)) g^{\prime}(t) d t=h \sum_{j=-N}^{N} w_{j} f\left(x_{j}\right)+E(h),
$$

where $x_{j}=g(h j)$ and $w_{j}=g^{\prime}(h j)$, and where $N$ is chosen large enough that $\left|w_{j} f\left(x_{j}\right)\right|<\epsilon$ for $|j|>N$. Here $\epsilon=10^{-p}$, where $p$ is the numeric precision level in digits. Because $g^{\prime}(t)$ and higher derivatives tend to zero very rapidly for large $t$, the resulting integrand $f(g(t)) g^{\prime}(t)$ typically is a smooth bell-shaped function, even if $f(t)$ itself is not well-behaved at the endpoints. For such bell-shaped integrands on an infinite interval, the Euler-Maclaurin formula implies that the error $E(h)$ in (1) decreases faster than any power of $h$. Additional details are given in [12] and [20, pg. 312-313].

\section{Numerical Experimentation}

We next present several specific examples of applying these tools in mathematical research. This is but a brief summary, as literally hundreds of concrete examples could be provided. This review, parts of which are excerpted from other writings by the present authors, emphasizes some relatively new examples, which have only recently been published, or (in at least one case) appear here for the first time.

One application of a high-precision quadrature facility, together with a PSLQ program, is to evaluate a definite integral in terms of a hypothesized set of analytic terms. As a first example, the present authors empirically discovered (then rigorously determined) that

$$
\begin{aligned}
\frac{2}{\sqrt{3}} \int_{0}^{1} \frac{\ln ^{6}(x) \arctan [x \sqrt{3} /(x-2)]}{x+1} d x=\frac{1}{81648}\left[-229635 L_{-3}(8)\right. \\
\quad+29852550 L_{-3}(7) \ln 3-1632960 L_{-3}(6) \pi^{2}+27760320 L_{-3}(5) \zeta(3) \\
\quad-275184 L_{-3}(4) \pi^{4}+36288000 L_{-3}(3) \zeta(5)-30008 L_{-3}(2) \pi^{6} \\
\left.\quad-57030120 L_{-3}(1) \zeta(7)\right],
\end{aligned}
$$

where

$$
L_{-3}(s):=\sum_{n=1}^{\infty}\left\{\frac{1}{(3 n-2)^{s}}-\frac{1}{(3 n-1)^{s}}\right\} .
$$

More general results have been conjectured, but (as often is the case), as of yet they are not all rigorously established [6].

3.1. A Monthly problem. In a very recent application of these methods, the authors addressed problem 11275 in the February 2007 issue of the American Mathematical Monthly [14], which problem asks to evaluate the iterated integral

$$
G:=\int_{0}^{\infty} \int_{y}^{\infty} \frac{(x-y)^{2} \log ((x+y) /(x-y))}{x y \sinh (x+y)} d x d y
$$


When this issue of the Monthly arrived in the mail, both of the present authors recognized that this problem was amenable to experimental methods, and independently began to work on it. Bailey set out to calculate the original double integral, after making the minor substitution $u=x-y$, so that both integrals have constant limits. This effort produced the numerical result

$$
G=1.1532659890804730178602752931059938854511244009224435425100 \ldots
$$

Bailey tried using the Inverse Symbolic Calculator (ISC), an online numeric constant recognition tool available at http://oldweb.cecm.sfu.ca/projects/ISC/ISCmain.html, but it was not able to recognize this constant.

Meanwhile, Borwein, working in a Maple environment, employed the simple substitution $x=t y$ to transform the integral into

$$
G=\int_{0}^{\infty} \int_{1}^{\infty} \frac{y(t-1)^{2} \log ((t+1) /(t-1))}{t \sinh (t y+y)} d t d y
$$

He then interchanged the order of integrals to produce the 1-D integral

$$
G=\frac{\pi^{2}}{4} \int_{1}^{\infty} \frac{(t-1)^{2}(\log (t+1)-\log (t-1))}{t(t+1)^{2}} d t
$$

which after the substitution $t=1 / s$ yielded

$$
G=\frac{\pi^{2}}{4} \int_{0}^{1} \frac{(s-1)^{2}(\log (1+s)-\log (1-s))}{s\left(1+s^{2}\right)} d s .
$$

Now Maple was able to numerically evaluate either form of the single integral integral (without the external coefficient) as $0.4674011002723397 \ldots$, and further was able to recognize this constant as $\pi^{2} / 4-2$, via the identify function (Mathematica has a RootApproximant operator, but it can only find algebraic numbers). Thus, the entire integral was recognized as

$$
G=\frac{\pi^{4}}{16}-\frac{\pi^{2}}{2}
$$

Now that Borwein "knew" the answer, it was a fairly simple matter, still working in a Maple environment, to "prove" it. This was done by substituting $u=(1-$ $s) /(1+s)$ in the third form above to yield the simple equivalent form

$$
G=\frac{2 \pi^{2}}{4} \int_{0}^{1} \frac{u^{2} \log u}{u^{2}-1} d u
$$

which Maple was able to evaluate analytically to produce the closed-form result, and which one can do by hand-say by using the geometric series and integrating term-by-term.

It was disappointing that the ISC was not able to recognize the numerical value of the original integral-evidently this constant lies just outside the search space and stored values that it works with. Over the summer of 2007 a revised and enhanced, parallel utility came on line at http://glooscap.cs.dal.ca:8087/ that is able to produce the closed-form evaluation for this problem, using the original numerical value as input. Indeed, the single Maple instruction identify ( 1.15326598908047301786027 ,BasisSizePoly=7); immediately returns (3). 


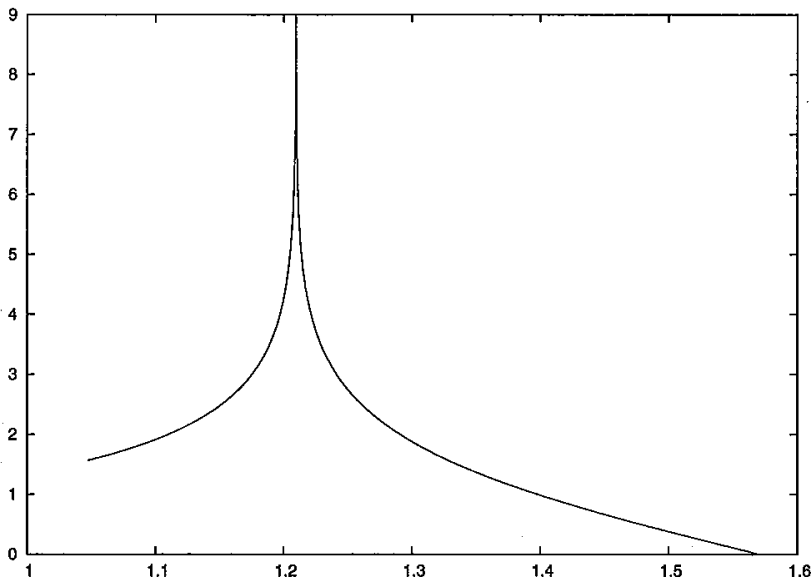

FIgURE 2. Plot of integrand function with singularity

3.2. A quantum field theory integral. Another result is the following, which is equivalent to one found by one of the present authors (Borwein) and British physicist David Broadhurst [23]:

$$
\begin{aligned}
& \text { (4) } \frac{24}{7 \sqrt{7}} \int_{\pi / 3}^{\pi / 2} \ln \left|\frac{\tan t+\sqrt{ } 7}{\tan t-\sqrt{7}}\right| d t \stackrel{?}{=} L_{-7}(2)= \\
& \sum_{n=0}^{\infty}\left[\frac{1}{(7 n+1)^{2}}+\frac{1}{(7 n+2)^{2}}-\frac{1}{(7 n+3)^{2}}+\frac{1}{(7 n+4)^{2}}-\frac{1}{(7 n+5)^{2}}-\frac{1}{(7 n+6)^{2}}\right] .
\end{aligned}
$$

This integral arose out of some studies in quantum field theory, in analysis of the volume of ideal tetrahedra in hyperbolic space. It is the simplest of 998 empirically determined cases where the volume of a hyperbolic knot complement is expressible in terms of an $L$-series and an apparently unexpected integral or sum [23]. The question mark is used here because although this identity has been numerically verified to 20,000-digit precision, as of this date no proof is yet known. Note that the integrand function has a nasty singularity (see Figure 2). This integral was computed using the tanh-sinh quadrature scheme after first splitting the integral into two parts- the first from $\pi / 3$ to $\tan ^{-1} \sqrt{7}$ (the singularity point), and the second from $\tan ^{-1} \sqrt{7}$ to $\pi / 2$.

This computation was performed on 1024 CPUs of the Apple G5 Terascale Computing Facility at Virginia Tech. By utilizing some advanced techniques to ensure an even computational load among processors, an almost perfect parallel speedup (993 out of 1024) was ultimately achieved (see Table 1).

PSLQ computations were further able to recover relations among integrals of this type. Let $I_{n}$ be the definite integral of (4), except with limits $n \pi / 24$ and $(n+1) \pi / 24$. Then the relations

$$
\begin{array}{rll}
-2 I_{2}-2 I_{3}-2 I_{4}-2 I_{5}+I_{8}+I_{9}-I_{10}-I_{11} & \stackrel{?}{=} & 0 \\
I_{2}+3 I_{3}+3 I_{4}+3 I_{5}+2 I_{6}+2 I_{7}-3 I_{8}-I_{9} & \stackrel{?}{=} & 0
\end{array}
$$




\begin{tabular}{|r|r|r|r|r|r|}
\hline CPUs & Init & Integral \#1 & Integral \#2 & Total & Speedup \\
\hline 1 & 190013 & 1534652 & 1026692 & 2751357 & 1.00 \\
16 & 12266 & 101647 & 64720 & 178633 & 15.40 \\
64 & 3022 & 24771 & 16586 & 44379 & 62.00 \\
256 & 770 & 6333 & 4194 & 11297 & 243.55 \\
1024 & 199 & 1536 & 1034 & 2769 & 993.63 \\
\hline
\end{tabular}

TABLE 1. Run times in seconds for the quadrature in equation (4)

have been numerically discovered, although as before we have no hint of how to prove this conjecture.

3.3. Ising integrals. Recently the present authors, together with Richard Crandall, were studying the following classes of integrals, which are related to Ising theory of mathematical physics:

$$
\begin{aligned}
C_{n} & :=\frac{4}{n !} \int_{0}^{\infty} \cdots \int_{0}^{\infty} \frac{1}{\left(\sum_{j=1}^{n}\left(u_{j}+1 / u_{j}\right)\right)^{2}} \frac{d u_{1}}{u_{1}} \cdots \frac{d u_{n}}{u_{n}} \\
D_{n} & :=\frac{4}{n !} \int_{0}^{\infty} \cdots \int_{0}^{\infty} \frac{\prod_{i<j}\left(\frac{u_{i}-u_{j}}{u_{i}+u_{j}}\right)^{2}}{\left(\sum_{j=1}^{n}\left(u_{j}+1 / u_{j}\right)\right)^{2}} \frac{d u_{1}}{u_{1}} \cdots \frac{d u_{n}}{u_{n}} .
\end{aligned}
$$

Consider the simplex $u_{1}>u_{2}>\cdots>u_{n}$. We change variables via $u_{k}:=\prod_{i=1}^{k} t_{i}$, with $t_{1} \in(0, \infty)$ and other $t_{i} \in(0,1)$. Define

$$
w_{k}:=\prod_{i=2}^{k} t_{i}, \quad v_{k}:=\prod_{i=k}^{n} t_{i} .
$$

and the functions

$$
\begin{aligned}
& \mathcal{A}_{n}\left(t_{2}, t_{3} \ldots, t_{n}\right):=\left(\prod_{n \geq k>j \geq 1} \frac{u_{k} / u_{j}-1}{u_{k} / u_{j}+1}\right)^{2} \\
& \mathcal{B}_{n}\left(t_{2}, t_{3} \ldots, t_{n}\right):=\frac{1}{\left(1+\sum_{k=2}^{n} w_{k}\right)\left(1+\sum_{k=2}^{n} v_{k}\right)} .
\end{aligned}
$$

Then the integrals can be recast as:

$$
\begin{aligned}
D_{n} & =2 \int_{0}^{1} \cdots \int_{0}^{1} \mathcal{A} \mathcal{B} d t_{2} d t_{3} \cdots d t_{n}, \\
C_{n} & =2 \int_{0}^{1} \cdots \int_{0}^{1} \mathcal{B} d t_{2} d t_{3} \cdots d t_{n}
\end{aligned}
$$

Here, the $1 / n$ ! has disappeared due to the $n$ ! ways of ordering the simplex indices, and we have symbolically integrated over $t_{1}$. It turned out to be useful to define

$$
E_{n}:=2 \int_{0}^{1} \cdots \int_{0}^{1} \mathcal{A} d t_{2} d t_{3} \cdots d t_{n}
$$

At present, there is no known practical scheme to find multi-hundred-digit values of general iterated integrals, except for straightforward extensions of 1-D 
schemes, and these become impractically expensive beyond 3-D. However, we observed that in the case of the $C_{n}$ integrals, we have

$$
C_{n}=\frac{2^{n}}{n !} \int_{0}^{\infty} t K_{0}^{n}(t) d t
$$

where $K_{0}(t)$ denotes the modified Bessel function [1]. This "trick" converts the problem to a 1-D integral that is amenable to high-precision quadrature using (for instance) the tanh-sinh quadrature scheme, thus dramatically reducing the computational cost.

We then computed high-precision values (up to 1000 digits) of $C_{n}$ for selected values of $n$, ranging from 3 up to 1024 (corresponding to a 1024-fold iterated integral). With these numerical values in hand, we were able to find - via PSLQ - and then prove the following intriguing results [10]: $C_{3}=\mathrm{L}_{-3}(2)$, with notation as in $(2)$, and $C_{4}=14 \zeta(3)$.

Along this line, we noticed that for large $n$, the numerical values appear to approach a constant, e.g.,

$$
\begin{aligned}
C_{10} & =0.63188002414701222229035087366080283 \ldots \\
C_{40} & =0.63047350337836353186994190185909694 \ldots \\
C_{100} & =0.63047350337438679612204019271903171 \ldots \\
C_{200} & =0.63047350337438679612204019271087890 \ldots
\end{aligned}
$$

What is this numerical value? By using the Inverse Symbolic Calculator tool (mentioned above in Section 3.1), we immediately found our answer:

$$
\lim _{n \rightarrow \infty} C_{n}=2 e^{-2 \gamma}
$$

where $\gamma$ is Euler's constant. We subsequently proved this intriguing numerical discovery [10], and in doing so discovered the fundamental nature of the Bessel function representation used in (9). Further research, experimental and theoretical, established the following results:

$$
\begin{aligned}
D_{2}= & 1 / 3, \quad D_{3}=8+4 \pi^{2} / 3-27 \mathrm{~L}_{-3}(2) \\
D_{4}= & 4 \pi^{2} / 9-1 / 6-7 \zeta(3) / 2 \\
E_{2}= & 6-8 \log 2 \\
E_{3}= & 10-2 \pi^{2}-8 \log 2+32 \log ^{2} 2 \\
E_{4}= & 22-82 \zeta(3)-24 \log 2+176 \log ^{2} 2-256\left(\log ^{3} 2\right) / 3 \\
& +16 \pi^{2} \log 2-22 \pi^{2} / 3 \\
E_{5} \stackrel{?}{=} & 42-1984 \mathrm{Li}_{4}(1 / 2)+189 \pi^{4} / 10-74 \zeta(3)-1272 \zeta(3) \log 2 \\
& +40 \pi^{2} \log ^{2} 2-62 \pi^{2} / 3+40\left(\pi^{2} \log 2\right) / 3+88 \log ^{4} 2 \\
& +464 \log ^{2} 2-40 \log 2
\end{aligned}
$$

(In the case of $D_{2}, D_{3}, D_{4}$, these results were already known by quite different methods.)

The result for $E_{5}$ required considerable effort, both computational and analytical. Although in this case we were not able to reduce the integral to a 1-D form, we were nonetheless able to reduce its dimension once more. The stultifying 3-D integral that resulted from this manipulation is shown in Table 2. As you might 
imagine, just converting this expression (originally produced as a Mathematica expression) to a working computer program required considerable effort. The final numerical evaluation of this integral required four hours on 64 CPUs of the Virginia Tech Apple system. Applying PSLQ to the resulting numerical value (together with the numerical values of a set of conjectured terms), yielded the experimental evaluation shown above. By the way, the plot shown in Figure 1 is for the PSLQ run that discovered this identity.

Like all good research problems, some generalizations are even more intriguing. To that end, we also studied the doubly indexed integrals [7]:

$$
C_{n, k}:=\frac{4}{n !} \int_{0}^{\infty} \cdots \int_{0}^{\infty} \frac{1}{\left(\sum_{j=1}^{n}\left(u_{j}+1 / u_{j}\right)\right)^{k+1}} \frac{d u_{1}}{u_{1}} \cdots \frac{d u_{n}}{u_{n}}
$$

The $C_{n, k}$ happily can be written in a form like (9). We first computed 1000-digit numerical values for all $n$ up to 36 and all $k$ up to 75 - a total of 2660 individual quadrature calculations, which were performed independently on a highly parallel computer system. Using PSLQ, we then discovered linear relations in each of the rows of this array. Some low-degree examples of these relations are the following:

$$
\begin{aligned}
& 0=C_{3,0}-84 C_{3,2}+216 C_{3,4} \\
& 0=2 C_{3,1}-69 C_{3,3}+135 C_{3,5} \\
& 0=C_{3,2}-24 C_{3,4}+40 C_{3,6} \\
& 0=32 C_{3,3}-630 C_{3,5}+945 C_{3,7} \\
& 0=125 C_{3,4}-2172 C_{3,6}+3024 C_{3,8} .
\end{aligned}
$$

These relations become rather complicated for large $n$. For $n=24$, for example, we found

$$
\begin{aligned}
0 \stackrel{?}{=} & C_{24,1} \\
& -1107296298 C_{24,3} \\
& +1288574336175660 C_{24,5} \\
& -88962910652291256000 C_{24,7} \\
& +1211528914846561331193600 C_{24,9} \\
& -5367185923241422152980553600 C_{24,11} \\
& +9857686103738772925980190636800 C_{24,13} \\
& -8476778037073141951236532459008000 C_{24,15} \\
& +3590120926882411593645052529049600000 C_{24,17} \\
& -745759114781380983188217871663104000000 C_{24,19} \\
& +71215552121869985477578381170258739200000 C_{24,21} \\
& -2649853457247995406113355087174696960000000 C_{24,23} \\
& +24912519234220575094208313195233280000000000 C_{24,25}
\end{aligned}
$$




$$
\begin{aligned}
& E_{5}=\int_{0}^{1} \int_{0}^{1} \int_{0}^{1}\left[2(1-x)^{2}(1-y)^{2}(1-x y)^{2}(1-z)^{2}(1-y z)^{2}(1-x y z)^{2}\right. \\
& \left(-\left[4 ( x + 1 ) ( x y + 1 ) \operatorname { l o g } ( 2 ) \left(y^{5} z^{3} x^{7}-y^{4} z^{2}(4(y+1) z+3) x^{6}-y^{3} z\left(\left(y^{2}+1\right) z^{2}+4(y+\right.\right.\right.\right. \\
& \text { 1) } z+5) x^{5}+y^{2}\left(4 y(y+1) z^{3}+3\left(y^{2}+1\right) z^{2}+4(y+1) z-1\right) x^{4}+y\left(z \left(z^{2}+4 z\right.\right. \\
& \left.+5) y^{2}+4\left(z^{2}+1\right) y+5 z+4\right) x^{3}+\left(\left(-3 z^{2}-4 z+1\right) y^{2}-4 z y+1\right) x^{2}-(y(5 z+4) \\
& +4) x-1)] /\left[(x-1)^{3}(x y-1)^{3}(x y z-1)^{3}\right]+\left[3(y-1)^{2} y^{4}(z-1)^{2} z^{2}(y z\right. \\
& -1)^{2} x^{6}+2 y^{3} z\left(3(z-1)^{2} z^{3} y^{5}+z^{2}\left(5 z^{3}+3 z^{2}+3 z+5\right) y^{4}+(z-1)^{2} z\right. \\
& \left(5 z^{2}+16 z+5\right) y^{3}+\left(3 z^{5}+3 z^{4}-22 z^{3}-22 z^{2}+3 z+3\right) y^{2}+3\left(-2 z^{4}+z^{3}+2\right. \\
& \left.\left.z^{2}+z-2\right) y+3 z^{3}+5 z^{2}+5 z+3\right) x^{5}+y^{2}\left(7(z-1)^{2} z^{4} y^{6}-2 z^{3}\left(z^{3}+15 z^{2}\right.\right. \\
& +15 z+1) y^{5}+2 z^{2}\left(-21 z^{4}+6 z^{3}+14 z^{2}+6 z-21\right) y^{4}-2 z\left(z^{5}-6 z^{4}-27 z^{3}\right. \\
& \left.-27 z^{2}-6 z+1\right) y^{3}+\left(7 z^{6}-30 z^{5}+28 z^{4}+54 z^{3}+28 z^{2}-30 z+7\right) y^{2}-2\left(7 z^{5}\right. \\
& \left.\left.+15 z^{4}-6 z^{3}-6 z^{2}+15 z+7\right) y+7 z^{4}-2 z^{3}-42 z^{2}-2 z+7\right) x^{4}-2 y\left(z ^ { 3 } \left(z^{3}\right.\right. \\
& \left.-9 z^{2}-9 z+1\right) y^{6}+z^{2}\left(7 z^{4}-14 z^{3}-18 z^{2}-14 z+7\right) y^{5}+z\left(7 z^{5}+14 z^{4}+3\right. \\
& \left.z^{3}+3 z^{2}+14 z+7\right) y^{4}+\left(z^{6}-14 z^{5}+3 z^{4}+84 z^{3}+3 z^{2}-14 z+1\right) y^{3}-3\left(3 z^{5}\right. \\
& \left.+6 z^{4}-z^{3}-z^{2}+6 z+3\right) y^{2}-\left(9 z^{4}+14 z^{3}-14 z^{2}+14 z+9\right) y+z^{3}+7 z^{2}+7 z \\
& +1) x^{3}+\left(z^{2}\left(11 z^{4}+6 z^{3}-66 z^{2}+6 z+11\right) y^{6}+2 z\left(5 z^{5}+13 z^{4}-2 z^{3}-2 z^{2}\right.\right. \\
& +13 z+5) y^{5}+\left(11 z^{6}+26 z^{5}+44 z^{4}-66 z^{3}+44 z^{2}+26 z+11\right) y^{4}+\left(6 z^{5}-4\right. \\
& \left.z^{4}-66 z^{3}-66 z^{2}-4 z+6\right) y^{3}-2\left(33 z^{4}+2 z^{3}-22 z^{2}+2 z+33\right) y^{2}+\left(6 z^{3}+26\right. \\
& \left.\left.z^{2}+26 z+6\right) y+11 z^{2}+10 z+11\right) x^{2}-2\left(z^{2}\left(5 z^{3}+3 z^{2}+3 z+5\right) y^{5}+z\left(22 z^{4}\right.\right. \\
& \left.+5 z^{3}-22 z^{2}+5 z+22\right) y^{4}+\left(5 z^{5}+5 z^{4}-26 z^{3}-26 z^{2}+5 z+5\right) y^{3}+\left(3 z^{4}-\right. \\
& \left.\left.22 z^{3}-26 z^{2}-22 z+3\right) y^{2}+\left(3 z^{3}+5 z^{2}+5 z+3\right) y+5 z^{2}+22 z+5\right) x+15 z^{2}+2 z \\
& +2 y(z-1)^{2}(z+1)+2 y^{3}(z-1)^{2} z(z+1)+y^{4} z^{2}\left(15 z^{2}+2 z+15\right)+y^{2}\left(15 z^{4}\right. \\
& \left.\left.-2 z^{3}-90 z^{2}-2 z+15\right)+15\right] /\left[(x-1)^{2}(y-1)^{2}(x y-1)^{2}(z-1)^{2}(y z-1)^{2}\right. \\
& \left.(x y z-1)^{2}\right]-\left[4 ( x + 1 ) ( y + 1 ) ( y z + 1 ) \left(-z^{2} y^{4}+4 z(z+1) y^{3}+\left(z^{2}+1\right) y^{2}\right.\right. \\
& -4(z+1) y+4 x\left(y^{2}-1\right)\left(y^{2} z^{2}-1\right)+x^{2}\left(z^{2} y^{4}-4 z(z+1) y^{3}-\left(z^{2}+1\right) y^{2}\right. \\
& +4(z+1) y+1)-1) \log (x+1)] /\left[(x-1)^{3} x(y-1)^{3}(y z-1)^{3}\right]-[4(y+1)(x y \\
& +1)(z+1)\left(x^{2}\left(z^{2}-4 z-1\right) y^{4}+4 x(x+1)\left(z^{2}-1\right) y^{3}-\left(x^{2}+1\right)\left(z^{2}-4 z-1\right)\right. \\
& \left.\left.y^{2}-4(x+1)\left(z^{2}-1\right) y+z^{2}-4 z-1\right) \log (x y+1)\right] /\left[x(y-1)^{3} y(x y-1)^{3}(z-\right. \\
& \left.1)^{3}\right]-\left[4 ( z + 1 ) ( y z + 1 ) \left(x^{3} y^{5} z^{7}+x^{2} y^{4}(4 x(y+1)+5) z^{6}-x y^{3}\left(\left(y^{2}+\right.\right.\right.\right. \\
& \text { 1) } \left.x^{2}-4(y+1) x-3\right) z^{5}-y^{2}\left(4 y(y+1) x^{3}+5\left(y^{2}+1\right) x^{2}+4(y+1) x+1\right) z^{4}+ \\
& y\left(y^{2} x^{3}-4 y(y+1) x^{2}-3\left(y^{2}+1\right) x-4(y+1)\right) z^{3}+\left(5 x^{2} y^{2}+y^{2}+4 x(y+1)\right. \\
& \left.\left.\left.\left.y+1) z^{2}+((3 x+4) y+4) z-1\right) \log (x y z+1)\right] /\left[x y(z-1)^{3} z(y z-1)^{3}(x y z-1)^{3}\right]\right)\right] \\
& /\left[(x+1)^{2}(y+1)^{2}(x y+1)^{2}(z+1)^{2}(y z+1)^{2}(x y z+1)^{2}\right] d x d y d z
\end{aligned}
$$

TABLE 2. The $E_{5}$ integral 
By performing some additional analysis on the coefficients of these empirical relations, we were then led to the following general formulas:

$$
\begin{aligned}
0= & (k+1) C_{1, k}-(k+2) C_{1, k+2} \\
0= & (k+1)^{2} C_{2, k}-4(k+2)^{2} C_{2, k+2} \\
0= & (k+1)^{3} C_{3, k}-2(k+2)\left(5(k+2)^{2}+1\right) C_{3, k+2} \\
& +9(k+2)(k+3)(k+4) C_{3, k+4} \\
0= & (k+1)^{4} C_{4, k}-4(k+2)^{2}\left(5(k+2)^{2}+3\right) C_{4, k+2} \\
& +64(k+2)(k+3)^{2}(k+4) C_{4, k+4} \\
0 \stackrel{?}{=} & (k+1)^{5} C_{5, k}-(k+2)\left(35 k^{4}+280 k^{3}+882 k^{2}+1288 k+731\right) C_{5, k+2} \\
& +(k+2)(k+3)(k+4)\left(259 k^{2}+1554 k+2435\right) C_{5, k+4} \\
& -225(k+2)(k+3)(k+4)(k+5)(k+6) C_{5, k+6} \\
0 & (k+1)^{6} C_{6, k}-8(k+2)^{2}\left(7 k^{4}+56 k^{3}+182 k^{2}+280 k+171\right) C_{6, k+2} \\
& +16(k+2)(k+3)^{2}(k+4)\left(49 k^{2}+294 k+500\right) C_{6, k+4} \\
& -2304(k+2)(k+3)(k+4)^{2}(k+5)(k+6) C_{6, k+6}
\end{aligned}
$$

where again the question mark is used to indicate relations that were originally not yet formally established. The original proofs of the first two are easy; the second two are harder and relied on WZ techniques of the type discussed in Section 4 (see also [7]).

In this analysis, we found it useful to define $c_{n, k}=n ! k ! 2^{-n} C_{n, k}$. Let $M$ be the largest integer in $(n+1) / 2$. We found (using extensive high-precision polynomial regression) that all of these recursions can be written in the compact form

$$
\sum_{i=0}^{M}(-1)^{i} p_{n, i}(k+i+1) c_{n, k+2 i}=0
$$

for certain relatively simple polynomials $p_{n, i}(x)$. Here are the polynomials for $n=5$ and $n=6$ :

$$
\begin{array}{ll}
p_{5,0}(x)=x^{6} & p_{6,0}(x)=x^{7} \\
p_{5,1}(x)=35 x^{4}+42 x^{2}+3 & p_{6,1}(x)=x\left(56 x^{4}+112 x^{2}+24\right) \\
p_{5,2}(x)=259 x^{2}+104 & p_{6,2}(x)=x\left(784 x^{2}+944\right) \\
p_{5,3}(x)=225 & p_{6,3}(x)=2304 x
\end{array}
$$

Thus we had uncovered one more layer of this very interesting "onion" problem that as yet defies complete understanding. A complete constructive proof of such recursions is now available in [26].

3.4. Heisenberg spin integrals. Beginning in late 2006 we investigated the following integrals ("spin integrals"), which arise, like the Ising integrals, from studies in mathematical physics [15]:

$$
\begin{aligned}
P(n):= & \frac{\pi^{n(n+1) / 2}}{(2 \pi i)^{n}} \cdot \int_{-\infty}^{\infty} \int_{-\infty}^{\infty} \cdots \int_{-\infty}^{\infty} U\left(x_{1}-i / 2, x_{2}-i / 2, \cdots, x_{n}-i / 2\right) \\
& \times T\left(x_{1}-i / 2, x_{2}-i / 2, \cdots, x_{n}-i / 2\right) d x_{1} d x_{2} \cdots d x_{n}
\end{aligned}
$$


where

$$
\begin{aligned}
U\left(x_{1}-i / 2, x_{2}-i / 2, \cdots, x_{n}-i / 2\right) & =\frac{\prod_{1 \leq k<j \leq n} \sinh \left[\pi\left(x_{j}-x_{k}\right)\right]}{\prod_{1 \leq j \leq n} i^{n} \cosh ^{n}\left(\pi x_{j}\right)} \\
T\left(x_{1}-i / 2, x_{2}-i / 2, \cdots, x_{n}-i / 2\right) & =\frac{\prod_{1 \leq j \leq n}\left(x_{j}-i / 2\right)^{j-1}\left(x_{j}+i / 2\right)^{n-j}}{\prod_{1 \leq k<j \leq n}\left(x_{j}-x_{k}-i\right)} .
\end{aligned}
$$

Note that these integrals involve some complex-arithmetic calculations, even though the final results are real. So far we have been able to numerically confirm the following results:

$$
\begin{aligned}
P(1)= & \frac{1}{2} \\
P(2)= & \frac{1}{3}-\frac{1}{3} \log 2 \\
P(3)= & \frac{1}{4}-\log 2+\frac{3}{8} \zeta(3) \\
P(4)= & \frac{1}{5}-2 \log 2+\frac{173}{60} \zeta(3)-\frac{11}{6} \zeta(3) \log 2-\frac{51}{80} \zeta^{2}(3)-\frac{55}{24} \zeta(5)+\frac{85}{24} \zeta(5) \log 2 \\
P(5)= & \frac{1}{6}-\frac{10}{3} \log 2+\frac{281}{24} \zeta(3)-\frac{45}{2} \zeta(3) \log 2-\frac{489}{16} \zeta^{2}(3)-\frac{6775}{192} \zeta(5) \\
& +\frac{1225}{6} \zeta(5) \log 2-\frac{425}{64} \zeta(3) \zeta(5)-\frac{12125}{256} \zeta^{2}(5)+\frac{6223}{256} \zeta(7) \\
& -\frac{11515}{64} \zeta(7) \log 2+\frac{42777}{512} \zeta(3) \zeta(7),
\end{aligned}
$$

as well as a significantly more complicated expression for $P(6)$. We confirmed $P(1)$ through $P(4)$ to over 60 -digit precision; $P(5)$ to 30 -digit precision; and $P(6)$ to 8digit precision. These quadrature calculations were performed by means of parallel jobs on the Apple G5 cluster at Virginia Tech.

Boos and Korepin in [15] and later papers, using a variety of techniques from mathematical physics, conjecture that all $P(n)$ have the form above, namely as sums of products of alternating zeta functions. The evidence, while attractive, is not overwhelming, since although it seems very likely $P(n)$ is always expressible in terms of multi-zeta values (MZVs), as discussed in [20, Chap. 3], for weights of eight or higher these MZVs need not reduce to products of classical zeta functions. We hope that our direct methods will ultimately be able to confirm or confound the conjecture, although these computations are a daunting computational challenge due to their high-dimensional nature. In general, these experiences point to the critical need to develop better schemes for high-precision multi-dimensional integrals. The run times and processors used shown in the Table below underscore the rapidly escalating difficulty of these computations:

\begin{tabular}{|r|r|r|r|}
\hline$n$ & Digits & Processors & Run Time \\
\hline 2 & 120 & 1 & $10 \mathrm{sec}$. \\
3 & 120 & 8 & $55 \mathrm{~min}$. \\
4 & 60 & 64 & $27 \mathrm{~min}$. \\
5 & 30 & 256 & $39 \mathrm{~min}$. \\
6 & 6 & 256 & $59 \mathrm{hrs}$. \\
\hline
\end{tabular}

3.5. Ramanujan-type series. Some new types of infinite series formulas, based on elliptic integral approximations, were discovered by Ramanujan around 
1910, but these were not well known (nor fully proven) until quite recently when his writings were fully edited. They are based on elliptic functions and are described at length in [25]. One of these is the remarkable formula

$$
\frac{1}{\pi}=\frac{2 \sqrt{2}}{9801} \sum_{k=0}^{\infty} \frac{(4 k) !(1103+26390 k)}{(k !)^{4} 396^{4 k}}
$$

Each term of this series produces an additional eight correct digits in the result. Gosper used this formula to compute 17 million digits of $\pi$ in 1985 . At about the same time, David and Gregory Chudnovsky found the following variation of Ramanujan's formula:

$$
\frac{1}{\pi}=12 \sum_{k=0}^{\infty} \frac{(-1)^{k}(6 k) !(13591409+545140134 k)}{(3 k) !(k !)^{3} 640320^{3 k+3 / 2}}
$$

Each term of this series produces an additional 14 correct digits. They used this formula in several large calculations of $\pi$, culminating with in 1994 with a calculation to over four billion decimal digits. Their remarkable story was told in a prizewinning New Yorker article "The Mountains of Pi" [39].

In a related way, the Ramanujan-type series

$$
\frac{1}{\pi}=\sum_{n=0}^{\infty}\left(\frac{\left(\begin{array}{c}
2 n \\
n
\end{array}\right)}{16^{n}}\right)^{3} \frac{42 n+5}{16}
$$

allows one to compute the billionth binary digit of $1 / \pi$, or the like, without computing the first half of the series.

In some recent papers, J. Guillera has exhibited several new Ramanujan-style series formulas for reciprocal powers of $\pi$, including the following $[\mathbf{3 0}, \mathbf{3 2}, \mathbf{3 1}]$ :

$$
\begin{aligned}
\frac{128}{\pi^{2}} & =\sum_{n=0}^{\infty}(-1)^{n} r(n)^{5}\left(13+180 n+820 n^{2}\right)\left(\frac{1}{32}\right)^{2 n} \\
\frac{32}{\pi^{2}} & =\sum_{n=0}^{\infty}(-1)^{n} r(n)^{5}\left(1+8 n+20 n^{2}\right)\left(\frac{1}{2}\right)^{2 n} \\
\frac{32}{\pi^{3}} & =\sum_{n=0}^{\infty} r(n)^{7}\left(1+14 n+76 n^{2}+168 n^{3}\right)\left(\frac{1}{32}\right)^{2 n}
\end{aligned}
$$

where we define the function $r(n)$ as follows:

$$
r(n)=\frac{(1 / 2)_{n}}{n !}=\frac{1 / 2 \cdot 3 / 2 \cdots \cdots(2 n-1) / 2}{n !}=\frac{\Gamma(n+1 / 2)}{\sqrt{\pi} \Gamma(n+1)} .
$$

Guillera proved (13) and (14) using Wilf-Zeilberger's method described, which we will discuss more below. He ascribes series (15) to Gourevich who also found it using integer relation methods. Guillera also provides other series for $1 / \pi^{2}$ based on other Gamma function values as in (10) and (11) but for our experiments we restrict ourselves to series using $r(n)$. 
We have attempted to do a more thorough experimental search for identities of this general type. In particular, we searched for formulas of either of the two forms

$$
\begin{aligned}
\frac{c}{\pi^{m}} & =\sum_{n=0}^{\infty} r(n)^{2 m+1}\left(p_{0}+p_{1} n+\cdots+p_{m} n^{m}\right) \alpha^{2 n} \\
\frac{c}{\pi^{m}} & =\sum_{n=0}^{\infty}(-1)^{n} r(n)^{2 m+1}\left(p_{0}+p_{1} n+\cdots+p_{m} n^{m}\right) \alpha^{2 n} .
\end{aligned}
$$

Here $c$ is some integer linear combination of the constants $\left(d_{i}, 1 \leq i \leq 34\right)$ :

$$
\begin{aligned}
& 1,2^{1 / 2}, 2^{1 / 3}, 2^{1 / 4}, 2^{1 / 6}, 4^{1 / 3}, 8^{1 / 4}, 32^{1 / 6}, 3^{1 / 2}, 3^{1 / 3}, 3^{1 / 4}, 3^{1 / 6}, 9^{1 / 3}, \\
& 27^{1 / 4}, 243^{1 / 6}, 5^{1 / 2}, 5^{1 / 4}, 125^{1 / 4}, 7^{1 / 2}, 13^{1 / 2}, 6^{1 / 2}, 6^{1 / 3}, 6^{1 / 4}, 6^{1 / 6}, \\
& 7,36^{1 / 3}, 216^{1 / 4}, 7776^{1 / 6}, 12^{1 / 4}, 108^{1 / 4}, 10^{1 / 2}, 10^{1 / 4}, 15^{1 / 2} .
\end{aligned}
$$

The polynomial coefficients $\left(p_{k}, 1 \leq k \leq m\right)$ in (16) and (17) are each some integer linear combination of the constants $\left(q_{i}, 1 \leq i \leq 11\right)$ :

$$
1,2^{1 / 2}, 3^{1 / 2}, 5^{1 / 2}, 6^{1 / 2}, 7^{1 / 2}, 10^{1 / 2}, 13^{1 / 2}, 14^{1 / 2}, 15^{1 / 2}, 30^{1 / 2} .
$$

Note that the linear combination chosen for a given $p_{k}$ may be different from that chosen for any of the others. The constant $\alpha$ in (16) and (17) is chosen from

$$
\begin{aligned}
& 1 / 2,1 / 4,1 / 8,1 / 16,1 / 32,1 / 64,1 / 128,1 / 256, \sqrt{5}-2,(2-\sqrt{3})^{2}, \\
& 5 \sqrt{13}-18,(\sqrt{5}-1)^{4} / 128,(\sqrt{5}-2)^{4},\left(2^{1 / 3}-1\right)^{4} / 2,1 /(2 \sqrt{2}), \\
& (\sqrt{2}-1)^{2},(\sqrt{5}-2)^{2},(\sqrt{3}-\sqrt{2})^{4} .
\end{aligned}
$$

This list of $\alpha$ constants was taken from a table on page 172 of [25]. The need for this smorgasbord is that PSLQ cannot directly handle algebraic numbers, only integers; in effect we hard-wired a lot of choices into our hunt.

These searches were done using a two-level PSLQ integer relation finding program, with 1000-digit precision. Each selection of $m$ and $\alpha$ constituted'one separate integer relation search. In particular, for a fixed $m$ and $\alpha$ in (16), we calculated the $[34+11(m+1)]$-long set of real numbers

$$
\begin{aligned}
& d_{1}, d_{2}, \cdots, d_{34}, \\
& q_{0} \sum_{n=0}^{\infty} r(n)^{2 m+1} \alpha^{2 n}, q_{1} \sum_{n=0}^{\infty} r(n)^{2 m+1} \alpha^{2 n}, \cdots, q_{11} \sum_{n=0}^{\infty} r(n)^{2 m+1} \alpha^{2 n}, \\
& q_{1} \sum_{n=0}^{\infty} r(n)^{2 m+1} n \alpha^{2 n}, q_{2} \sum_{n=0}^{\infty} r(n)^{2 m+1} n \alpha^{2 n}, \cdots, q_{11} \sum_{n=0}^{\infty} r(n)^{2 m+1} n \alpha^{2 n}, \cdots \\
& q_{1} \sum_{n=0}^{\infty} r(n)^{2 m+1} n^{m} \alpha^{2 n}, q_{2} \sum_{n=0}^{\infty} r(n)^{2 m+1} n^{m} \alpha^{2 n}, \cdots, q_{11} \sum_{n=0}^{\infty} r(n)^{2 m+1} n^{m} \alpha^{2 n}
\end{aligned}
$$

and then applied a two-level PSLQ program, implemented using ARPREC multipleprecision software, to this vector.

After finding a relation with our program, we carefully checked to ensure that it was not reducible to another in the list by an algebraic manipulation. Also, in numerous cases, multiple relations existed. In such cases, we eliminated these one by one, typically by replacing one of the constants in the relation by an unrelated transcendental and re-running the program, until no additional relations were found. 
The result of this effort is the following list of relations. As it turns out, each of these are given either implicitly or explicitly in [25] or [32]. But just as important here is the apparent non-existence of any additional relations. In particular, if a relation is not shown below for a given $\alpha$ and/or sign choice, that means (as a consequence of our calculations) that there is no such relation with integer coefficients whose Euclidean norm is less than $10^{10}$. So in a very real sense, we had a splendid inductive experience in which we validated our methods and confirmed the null-hypothesis.

For degree $m=1$, with non-alternating signs:

$$
\begin{aligned}
\frac{4}{\pi} & =\sum_{n=0}^{\infty} r(n)^{3}(1+6 n)\left(\frac{1}{2}\right)^{2 n}, \\
\frac{16}{\pi} & =\sum_{n=0}^{\infty} r(n)^{3}(5+42 n)\left(\frac{1}{8}\right)^{2 n}, \\
\frac{12^{1 / 4}}{\pi} & =\sum_{n=0}^{\infty} r(n)^{3}(-15+9 \sqrt{3}-36 n+24 \sqrt{3} n)(2-\sqrt{3})^{4 n}, \\
\frac{32}{\pi} & =\sum_{n=0}^{\infty} r(n)^{3}(-1+5 \sqrt{5}+30 n+42 \sqrt{5} n)\left(\frac{(\sqrt{5}-1)^{4}}{128}\right)^{2 n}, \\
\frac{5^{1 / 4}}{\pi} & =\sum_{n=0}^{\infty} r(n)^{3}(-525+235 \sqrt{5}-1200 n+540 \sqrt{5} n)(\sqrt{5}-2)^{8 n} .
\end{aligned}
$$

For degree $m=1$, with alternating signs:

$$
\begin{aligned}
\frac{2 \sqrt{2}}{\pi} & =\sum_{n=0}^{\infty}(-1)^{n} r(n)^{3}(1+6 n)\left(\frac{1}{2 \sqrt{2}}\right)^{2 n} \\
\frac{2}{\pi} & =\sum_{n=0}^{\infty}(-1)^{n} r(n)^{3}(-5+4 \sqrt{2}-12 n+12 \sqrt{2} n)(\sqrt{2}-1)^{4 n} \\
\frac{2}{\pi} & =\sum_{n=0}^{\infty}(-1)^{n} r(n)^{3}(23-10 \sqrt{5}+60 n-24 \sqrt{5} n)(\sqrt{5}-2)^{4 n} \\
\frac{2}{\pi} & =\sum_{n=0}^{\infty}(-1)^{n} r(n)^{3}(177-72 \sqrt{6}+420 n-168 \sqrt{6} n)(\sqrt{3}-\sqrt{2})^{8 n} .
\end{aligned}
$$

For degree $m=2$,

$$
\begin{aligned}
\frac{8}{\pi^{2}} & =\sum_{n=0}^{\infty}(-1)^{n} r(n)^{5}\left(1+8 n+20 n^{2}\right)\left(\frac{1}{2}\right)^{2 n} \\
\frac{128}{\pi^{2}} & =\sum_{n=0}^{\infty}(-1)^{n} r(n)^{5}\left(13+180 n+820 n^{2}\right)\left(\frac{1}{32}\right)^{2 n}
\end{aligned}
$$

For degree $m=3$,

$$
\frac{32}{\pi^{3}}=\sum_{n=0}^{\infty} r(n)^{7}\left(1+14 n+76 n^{2}+168 n^{3}\right)\left(\frac{1}{8}\right)^{2 n}
$$


For degree $m=4,5$ we have been unable to find any similar series, with exclusion bounds roughly $10^{10}$ as before, thereby (so far) dashing our hope to find an infinite family of rational sums extending (12), (13), (14), and (15). More study, however, will certainly be needed to understand this phenomenon.

3.6. Elliptic and theta function theory. While (13), (14), (15) have no "explanation," there are tantalizing echoes of the elliptic theory, described in [25], which explains the series for $1 / \pi$ as we now partially reprise. We first define the theta functions $\theta_{3}, \theta_{4}$ and $\theta_{2}$

$$
\theta_{2}(q):=\sum_{n=-\infty}^{\infty} q^{(n+1 / 2)^{2}}, \quad \theta_{3}(q):=\sum_{n=-\infty}^{\infty} q^{n^{2}}, \quad \theta_{4}(q):=\sum_{n=-\infty}^{\infty}(-1)^{n} q^{n^{2}},
$$

for $|q|<1$. We next identify the invariant

$$
k_{N}=\frac{\theta_{2}^{2}}{\theta_{3}^{2}}\left(e^{-\pi \sqrt{N}}\right) \text {. }
$$

We denote the complementary modulus $k^{\prime}:=\sqrt{1-k^{2}}$ in terms of which it transpires that Jacobi's identity $\theta_{3}^{4}=\theta_{4}^{4}+\theta_{2}^{4}$ (see [25]) implies

$$
k_{N}^{\prime}=\frac{\theta_{4}^{2}}{\theta_{3}^{2}}\left(e^{-\pi \sqrt{N}}\right) \text {. }
$$

For reasons detailed in $[\mathbf{2 0}]$ and $[25]$ we know that for each natural number $N$, $k_{N}$ is algebraic. Thus, $k_{N}$ is a wonderful algebraic spray-gun as it is very easy to compute.

For example, $k_{1}=1 / \sqrt{2}=k_{1}^{\prime}$ while $k_{210}$ is the singular value sent to Hardy in Ramanujan's famous 1913 letters of introduction-ignored by two other famous English mathematicians:

$$
\begin{aligned}
k_{210}:= & (\sqrt{2}-1)^{2}(\sqrt{3}-2)(\sqrt{7}-6)^{2}(8-3 \sqrt{7}) \\
& \times(\sqrt{10}-3)^{2}(\sqrt{15}-\sqrt{14})(4-\sqrt{15})^{2}(6-\sqrt{35}) .
\end{aligned}
$$

Remarkably,

$$
k_{100}:=\left((3-2 \sqrt{2})(2+\sqrt{5})(-3+\sqrt{10})(-\sqrt{2}+\sqrt[4]{5})^{2}\right)^{2}
$$

arose in Bornemann's solution to Trefethen's 10th problem, $[\mathbf{1 7}, \mathbf{1 8}]$ : it arises in determining the probability that a Brownian motion starting at the center of a $10 \times 1$ box hits the ends first is $2 / \pi \arcsin \left(k_{100}\right)$. simpler

Ramanujan also noticed that the invariants $G_{N}$ and $g_{N}$ defined next are often

$$
G_{N}^{-12}:=2 k_{N} k_{N}^{\prime} \text { and } g_{N}^{-12}:=2 k_{N} / k_{N}^{\prime 2} .
$$

Note that each of these two latter invariants provides a quadratic formula for $k_{N}$. We also need Ramanujan's invariant of the second kind

$$
\alpha_{N}:=\frac{1 / \pi-q \theta_{4}^{\prime}(q) / \theta_{4}(q)}{\theta_{3}^{4}(q)} \quad q:=e^{-\pi \sqrt{N}}
$$


which is likewise algebraic for integer $N,[\mathbf{2 5}]$. In the form we have given them all the coefficients are very simple and quick to compute numerically. Hence integer relation methods are easy to apply.

3.6.1. Determining invariants. The following Maple code produces 20 digits of each of our invariants:

que : $=\mathrm{N}->\exp (-\mathrm{Pi} * \operatorname{sqrt}(\mathrm{N}))$ :

kk: $=q->(J a c o b i T h e t a 2(0, q) / J a c o b i T h e t a 3(0, q))^{\wedge} 2:$

$\mathrm{kc}:=\mathrm{q}->(\operatorname{JacobiTheta} 4(0, \mathrm{q}) / \operatorname{JacobiTheta3}(0, \mathrm{q}))^{\sim} 2: \mathrm{k}:=\mathrm{kk} @ q u e:$

l:=kc@que: $G:=1 /(2 * \mathrm{k} * 1): \mathrm{g}:=2 * \mathrm{k} / 1^{\wedge} 2$ :

alpha: $=r->(\operatorname{subs}(q=\exp (-P i * \operatorname{sqrt}(r))$,

$(1 / P i-\operatorname{sqrt}(r) * 4 *(q * \operatorname{diff}(\operatorname{JacobiTheta} 4(0, q), q) / \operatorname{JacobiTheta} 4(0, q))) /$

JacobiTheta3 $(0, q) \wedge 4))$ :

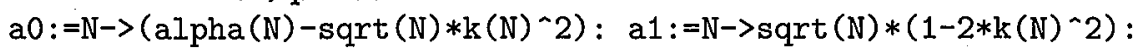

b0 : =N->alpha $(\mathrm{N}) /(1-\mathrm{k}(\mathrm{N}) \sim 2): \quad \mathrm{b} 1:=\mathrm{N} \rightarrow>\operatorname{sqrt}(\mathrm{N}) *\left(1+\mathrm{k}(\mathrm{N})^{\sim} 2\right) /\left(1-\mathrm{k}(\mathrm{N})^{\wedge} 2\right)$ :

We first explore use of Maple's identify function. Entering for $n$ to 6 do identify (evalf $[20](k(n)))$ od;

returns

$$
\begin{gathered}
1 / 2 \sqrt{2},-1+\sqrt{2}, 1 / 4 \sqrt{6}-1 / 4 \sqrt{2}, 3-2 \sqrt{2} \\
0.11887694580260010118,0.085164233174742587643 .
\end{gathered}
$$

where we have used only the simplest parameter-free version of the "identify" function. Correspondingly

for $n$ to 8 do identify (evalf $[20](G(2 * n-1))$ ) od;

returns for the first eight odd values of $G_{N}^{-12}$ :

$$
\begin{gathered}
1,2,2+\sqrt{5}, 8,7+4 \sqrt{3}, 4 / 3 \sqrt[3]{199+3 \sqrt{33}}+\frac{136}{3} \frac{1}{\sqrt[3]{199+3 \sqrt{33}}}+\frac{22}{3} \\
18+5 \sqrt{13}, 28+12 \sqrt{5}
\end{gathered}
$$

and

for $n$ to 8 do identify (evalf $[20](g(2 * n))$ ) od;

returns for the first five even values of $g_{N}^{-12}$ :

$$
1,1 / 4 \sqrt{2}, 3-2 \sqrt{2}, 1 / 4 \sqrt{-14+10 \sqrt{2}}, 9-4 \sqrt{5}
$$

but fails on the next three

\section{$0.034675177060507381314,0.022419012334044683484,0.014940167059400883091$}

This can be remedied in many ways. For example,

\section{_EnvExplicit:=true:}

(PolynomialTools [MinimalPolynomial] $\left.\left(g(14)^{-}(1 / 3), 4\right)\right)$;

solve $(\%)[2]$; evalf $\left(\% / g(14)^{\wedge}(1 / 3)\right)$;

yields $1-2 X-5 X^{2}-2 X^{3}+X^{4}$ as the polynomial potentially satisfied by $g_{14}^{-4}$; and then extracts the correct radical

$$
1 / 2+\sqrt{2}-1 / 2 \sqrt{5+4 \sqrt{2}}
$$

which is confirmed to 15 places. One may check that $\left(g_{14}^{6}+g_{14}^{-6}\right) / 2=\sqrt{2}+1$ is an even simpler invariant. Similarly, 
EnvExplicit: =true: (PolynomialTools [MinimalPolynomial]

$\left.\left(G(25)^{\wedge}(1 / 12), 4\right)\right)$;

illustrates that $G_{25}$ solves $x^{2}-x-1=0$ and so is the golden mean, and also shows that the appropriate power of $G_{N}, g_{N}$ varies with $N$. Armed with these tools a fine challenge is to obtain all values of $G_{N}, g_{N}$ or $k_{N}$ up to, say, $N=50$.

We may now record two families of series of which Ramanujan discovered many cases, see [25, p. 182]:

Theorem 1 (Reciprocal Series for Pi). (a) For integer $N>1$

$$
\frac{1}{\pi}=\sum_{n=0}^{\infty} r_{n}^{3}\left\{\left(\alpha_{N}-\sqrt{N} k_{N}^{2}\right)+n \sqrt{N}\left(k_{N}^{\prime 2}-k_{N}^{2}\right)\right\}\left(G_{N}^{-12}\right)^{2 n} .
$$

(b) For integer $N \geq 1$

$$
\frac{1}{\pi}=\sum_{n=0}^{\infty}(-1)^{n} r_{n}^{3}\left\{\alpha_{N} k_{N}^{\prime-2}+n \sqrt{N} \frac{1+k_{N}^{2}}{1-k_{N}^{2}}\right\}\left(g_{N}^{-12}\right)^{2 n} .
$$

3.6.2. Identifying our series. We shall now try to determine which cases of Theorem 1 we have recovered. Crude code to determine the coefficients is:

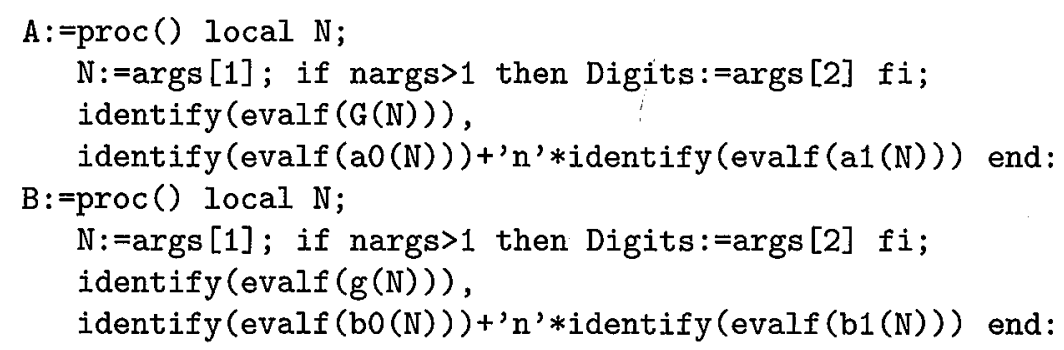

for the non-alternating and alternating cases respectively. For example, $B(1)$ returns $\sqrt{2}, 3 n+1 / 4$ which means that

$$
\frac{2}{\pi}=\sum_{n=0}^{\infty}(-1)^{n} r(n)^{3}(1+4 n) .
$$

We leave it to the reader to see that we had recovered the cases $N=3,5,7,15,25$ of (a) and $N=4,6,10,18$ of (b) in Theorem 1.

\section{Computer-Assisted Proof}

4.1. The Wilf-Zeilberger algorithm. One fascinating non-numerical algorithm is the Wilf-Zeilberger (WZ) algorithm, which employs "creative telescoping" to show that a sum (with either finitely or infinitely many terms) is zero. We will not provide here any details of this remarkable procedure, which is now available for public usage. Interested readers are referred to the very readable book by Petkovsek, Wilf and Zeilberger [38]. For most purposes, packages available in Maple and Mathematica suffice. Some other software implementing these schemes

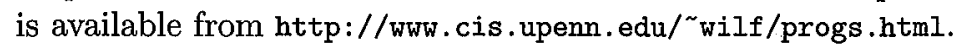


4.1.1. A first simple example. As a simple example of the usage of this scheme, we first present a WZ proof of the whimsical result $(1+1)^{n}=2^{n}$. This proof is from Doron Zeilberger's original Maple program, which in turn is inspired by the proof in $[43]$.

Let $F(n, k)=\left(\begin{array}{l}n \\ k\end{array}\right) 2^{-n}$. We wish to show that $L(n)=\sum_{k} F(n, k)=1$ for every $n$. To this end, we construct, using the WZ algorithm, the companion function

$$
G(n, k)=\frac{-1}{2^{(n+1)}}\left(\begin{array}{c}
n \\
k-1
\end{array}\right)\left(=\frac{-k}{2(n-k+1)} F(n, k)\right),
$$

and observe that

$$
F(n+1, k)-F(n, k)=G(n, k+1)-G(n, k) .
$$

By applying the obvious telescoping property of these functions, we can write

$$
\sum_{k} F(n+1, k)-\sum_{k} F(n, k)=\sum_{k}(G(n, k+1)-G(n, k))=0
$$

which establishes that $L(n+1)-L(n)=0$. The fact that $L(0)=1$ follows from the fact that $F(0, k)=1$ for $k=0$ and is 0 otherwise.

4.1.2. A second harder example. We will briefly present here the proof of the identities (13) and (14), which we repeat here in slightly different but equivalent forms:

$$
\begin{aligned}
\sum_{n=0}^{\infty} \frac{\left(\begin{array}{c}
4 n \\
2 n
\end{array}\right)\left(\begin{array}{c}
2 n \\
n
\end{array}\right)^{4}}{2^{16 n}}\left(120 n^{2}+34 n+3\right) & =\frac{32}{\pi^{2}} \\
\sum_{n=0}^{\infty} \frac{(-1)^{n}\left(\begin{array}{c}
2 n \\
n
\end{array}\right)^{5}}{2^{20 n}}\left(820 n^{2}+180 n+13\right) & =\frac{128}{\pi^{2}} .
\end{aligned}
$$

As we mentioned earlier, these can be and were discovered by a PSLQ-based search. strategy.

Guillera started by defining

$$
G(n, k)=\frac{(-1)^{k}}{2^{16 n} 2^{4 k}}\left(120 n^{2}+84 n k+34 n+10 k+3\right) \frac{\left(\begin{array}{c}
2 n \\
n
\end{array}\right)^{4}\left(\begin{array}{c}
2 k \\
k
\end{array}\right)^{3}\left(\begin{array}{c}
4 n-2 k \\
2 n-k
\end{array}\right)}{\left(\begin{array}{c}
2 n \\
k
\end{array}\right)\left(\begin{array}{c}
n+k \\
n
\end{array}\right)^{2}}
$$

He then used the software package $E K H A D$, which implements the WZ method, obtaining the companion formula

$$
F(n, k)=\frac{(-1)^{k} 512}{2^{16 n} 2^{4 k}} \frac{n^{3}}{4 n-2 k-1} \frac{\left(\begin{array}{c}
2 n \\
n
\end{array}\right)^{4}\left(\begin{array}{c}
2 k \\
k
\end{array}\right)^{3}\left(\begin{array}{c}
4 n-2 k \\
2 n-k
\end{array}\right)}{\left(\begin{array}{c}
2 n \\
k
\end{array}\right)\left(\begin{array}{c}
n+k \\
n
\end{array}\right)^{2}} .
$$

Zeilberger's theorem says that when we define

$$
H(n, k)=F(n+1, n+k)+G(n, n+k),
$$

then it follows that

$$
\sum_{n=0}^{\infty} G(n, 0)=\sum_{n=0}^{\infty} H(n, 0)
$$


which in this case is

$$
\begin{aligned}
\sum_{n=0}^{\infty} \frac{\left(\begin{array}{c}
2 n \\
n
\end{array}\right)^{4}\left(\begin{array}{c}
4 n \\
2 n
\end{array}\right)}{2^{16 n}}\left(120 n^{2}+34 n+3\right)= & \sum_{n=0}^{\infty} \frac{(-1)^{n}}{2^{20 n+7}} \frac{(n+1)^{3}}{2 n+3} \frac{\left(\begin{array}{c}
2 n+2 \\
n+1
\end{array}\right)^{4}\left(\begin{array}{c}
2 n \\
n
\end{array}\right)^{3}\left(\begin{array}{c}
2 n+4 \\
n+2
\end{array}\right)}{\left(\begin{array}{c}
2 n+2 \\
n
\end{array}\right)\left(\begin{array}{c}
2 n+1 \\
n+1
\end{array}\right)^{2}} \\
& +\sum_{n=0}^{\infty} \frac{(-1)^{n}}{2^{20 n}}\left(204 n^{2}+44 n+3\right)\left(\begin{array}{c}
2 n \\
n
\end{array}\right)^{5} \\
= & \frac{1}{4} \sum_{n=0}^{\infty} \frac{(-1)^{n}\left(\begin{array}{c}
2 n \\
n
\end{array}\right)^{5}}{2^{20 n}}\left(820 n^{2}+180 n+13\right)
\end{aligned}
$$

after considerable algebra.

Guillera then observes that since $\sum_{n \geq 0} G(n, k)=\sum_{n \geq 0} G(n, k+1)$, then by Carlson's theorem [13], it follows that $\sum_{n \geq 0} G(n, k)=A$ for some $A$, independent of $k$, even if $k$ is not an integer. We then note that $0<G(n, t) \leq 8^{-n}$, so one can interchange limit and sum to conclude that

$$
\lim _{t \rightarrow 1 / 2} \sum_{n=1}^{\infty} \operatorname{Re}[G(n, t)]=0 .
$$

Thus,

$$
A=\lim _{t \rightarrow 1 / 2} \operatorname{Re}[G(0, t)]=\frac{32}{\pi^{2}}
$$

and we have

$$
\sum_{n=0}^{\infty} G(n, k)=\sum_{n=0}^{\infty} H(n, k)=\frac{32}{\pi^{2}} .
$$

Guillera's two results follow immediately.

Obviously, this proof does not provide much insight, since the difficult part of the result is buried in the construction of (24). In other words, the WZ method provides "proofs," but these "proofs" tend to be relatively unenlightening. Nonetheless, the very general nature of this scheme is of interest; and the method, when it applies, does provide a certificate of its correctness.

It is worth noting that PSLQ and WZ complement each other very nicely: PSLQ is very useful for discovering new identities, but seldom provides any hint of how such results can be formally proved. WZ, on the other hand, is a powerful means to establish the truth of certain types of series identities, but it provides no suggestion as to how such identities could be found in the first place. The combination of these two methods presages a future in which a wide class of related results can be "discovered" and then "proved" automatically in a computer algebra system. Requisite details and additional applications of the WZ methodology are given in $[\mathbf{3 8}]$.

4.2. Apéry-like summations. Here we present a detailed case study in identifying sums of a certain class of infinite series, by means of a multi-step approach that is broadly illustrative of the experimental methodology in mathematics, and which involves the WZ method in a crucial way. The origins of this work lay in the existence of infinite series formulas involving central binomial coefficients in the denominators for the constants $\zeta(2), \zeta(3)$, and $\zeta(4)$. These formulas, as well the role of the formula for $\zeta(3)$ in Apéry's proof of its irrationality, have prompted 
considerable effort during the past few decades to extend these results to larger integer arguments. The formulas in question are

$$
\begin{aligned}
\zeta(2) & =3 \sum_{k=1}^{\infty} \frac{1}{k^{2}\left(\begin{array}{c}
2 k \\
k
\end{array}\right)} \\
\zeta(3) & =\frac{5}{2} \sum_{k=1}^{\infty} \frac{(-1)^{k+1}}{k^{3}\left(\begin{array}{c}
2 k \\
k
\end{array}\right)} \\
\zeta(4) & =\frac{36}{17} \sum_{k=1}^{\infty} \frac{1}{k^{4}\left(\begin{array}{c}
2 k \\
k
\end{array}\right)} .
\end{aligned}
$$

Identity (26) has been known since the 19th century, while (27) was variously discovered in the last century and (28) was noted by Comtet $[\mathbf{2 7}, \mathbf{2 4}, \mathbf{4 1}]$.

These results have led many to conjecture that the constant $\mathcal{Q}_{5}$ defined by the ratio

$$
\mathcal{Q}_{5}:=\zeta(5) / \sum_{k=1}^{\infty} \frac{(-1)^{k+1}}{k^{5}\left(\begin{array}{c}
2 k \\
k
\end{array}\right)}
$$

is rational, or at least algebraic. However, 10,000-digit PSLQ computations have established that if $\mathcal{Q}_{5}$ is a zero of a polynomial of degree at most 25 with integer coefficients, then the Euclidean norm of the vector of coefficients exceeds $1.24 \times 10^{383}$. Similar computations for $\zeta(5)$ have yielded a bound of $1.98 \times 10^{380}$. These computations lend substantial credence to the belief that $\mathcal{Q}_{5}$ and $\zeta(5)$ are transcendental.

Given the negative result from PSLQ computations for $\mathcal{Q}_{5}$, the authors of [21] systematically investigated the possibility of a multi-term identity of this general form for $\zeta(2 n+1)$. The following were recovered early in experimental searches using computer-based integer relation tools $[\mathbf{2 1 , 2 2}]$ :

$$
\begin{aligned}
& \zeta(5)=2 \sum_{k=1}^{\infty} \frac{(-1)^{k+1}}{k^{5}\left(\begin{array}{c}
2 k \\
k
\end{array}\right)}-\frac{5}{2} \sum_{k=1}^{\infty} \frac{(-1)^{k+1}}{k^{3}\left(\begin{array}{c}
2 k \\
k
\end{array}\right)} \sum_{j=1}^{k-1} \frac{1}{j^{2}} \\
& \zeta(7)=\frac{5}{2} \sum_{k=1}^{\infty} \frac{(-1)^{k+1}}{k^{7}\left(\begin{array}{c}
2 k \\
k
\end{array}\right)}+\frac{25}{2} \sum_{k=1}^{\infty} \frac{(-1)^{k+1}}{k^{3}\left(\begin{array}{c}
2 k \\
k
\end{array}\right)} \sum_{j=1}^{k-1} \frac{1}{j^{4}} \\
& \zeta(9)=\frac{9}{4} \sum_{k=1}^{\infty} \frac{(-1)^{k+1}}{k^{9}\left(\begin{array}{c}
2 k \\
k
\end{array}\right)}-\frac{5}{4} \sum_{k=1}^{\infty} \frac{(-1)^{k+1}}{k^{7}\left(\begin{array}{c}
2 k \\
k
\end{array}\right)} \sum_{j=1}^{k-1} \frac{1}{j^{2}}+5 \sum_{k=1}^{\infty} \frac{(-1)^{k+1}}{k^{5}\left(\begin{array}{c}
2 k \\
k
\end{array}\right)} \sum_{j=1}^{k-1} \frac{1}{j^{4}} \\
&+\frac{45}{4} \sum_{k=1}^{\infty} \frac{(-1)^{k+1}}{k^{3}\left(\begin{array}{c}
2 k \\
k
\end{array}\right)} \sum_{j=1}^{k-1} \frac{1}{j^{6}}-\frac{25}{4} \sum_{k=1}^{\infty} \frac{(-1)^{k+1}}{k^{3}\left(\begin{array}{c}
2 k \\
k
\end{array}\right)} \sum_{j=1}^{k-1} \frac{1}{j^{4}} \sum_{j=1}^{k-1} \frac{1}{j^{2}}, \\
& \zeta(11)=\frac{5}{2} \sum_{k=1}^{\infty} \frac{(-1)^{k+1}}{k^{11}\left(\begin{array}{c}
2 k \\
k
\end{array}\right)}+\frac{25}{2} \sum_{k=1}^{\infty} \frac{(-1)^{k+1}}{k^{7}\left(\begin{array}{c}
2 k \\
k
\end{array}\right)} \sum_{j=1}^{k-1} \frac{1}{j^{4}} \\
&-\frac{75}{4} \sum_{k=1}^{\infty} \frac{(-1)^{k+1}}{k^{3}\left(\begin{array}{c}
2 k \\
k
\end{array}\right)} \sum_{j=1}^{k-1} \frac{1}{j^{8}}+\frac{125}{4} \sum_{k=1}^{\infty} \frac{(-1)^{k+1}}{k^{3}\left(\begin{array}{c}
2 k \\
k
\end{array}\right)} \sum_{j=1}^{k-1} \frac{1}{j^{4}} \sum_{i=1}^{k-1} \frac{1}{i^{4}} .
\end{aligned}
$$


The general formula

$$
\sum_{k=1}^{\infty} \frac{1}{k\left(k^{2}-x^{2}\right)}=\frac{1}{2} \sum_{k=1}^{\infty} \frac{(-1)^{k+1}}{k^{3}\left(\begin{array}{c}
2 k \\
k
\end{array}\right)} \frac{5 k^{2}-x^{2}}{k^{2}-x^{2}} \prod_{m=1}^{k-1}\left(1-\frac{x^{2}}{m^{2}}\right)
$$

was obtained by Koecher [34] following techniques of Knopp and Schur.

Using bootstrapping and an application of the "Pade" function (which in both Mathematica and Maple produces Padé approximations to a rational function satisfied by a truncated power series), the following remarkable and unanticipated results were produced $[\mathbf{2 1}]$ :

$$
\sum_{k=1}^{\infty} \frac{1}{k^{3}\left(1-x^{4} / k^{4}\right)}=\frac{5}{2} \sum_{k=1}^{\infty} \frac{(-1)^{k+1}}{k^{3}\left(\begin{array}{c}
2 k \\
k
\end{array}\right)\left(1-x^{4} / k^{4}\right)} \prod_{m=1}^{k-1}\left(\frac{1+4 x^{4} / m^{4}}{1-x^{4} / m^{4}}\right)
$$

A decade later, following an analogous - but much more deliberate - experimentalbased procedure, as detailed below, we were able to provide a similar general formula for $\zeta(2 n+2)$ that is pleasingly parallel to (34). It is the following.

THeOREM 2 (Even Zeta Values, [9]). Let $x$ be a complex number not equal to a non-zero integer. Then

$$
\sum_{k=1}^{\infty} \frac{1}{k^{2}-x^{2}}=3 \sum_{k=1}^{\infty} \frac{1}{k^{2}\left(\begin{array}{c}
2 k \\
k
\end{array}\right)\left(1-x^{2} / k^{2}\right)} \prod_{m=1}^{k-1}\left(\frac{1-4 x^{2} / m^{2}}{1-x^{2} / m^{2}}\right) .
$$

Note that the left hand side of (35) is equal to

$$
\sum_{n=0}^{\infty} \zeta(2 n+2) x^{2 n}=\frac{1-\pi x \cot (\pi x)}{2 x^{2}} .
$$

Thus, (35) generates an Apéry-like formulae for $\zeta(2 n)$ for every positive integer $n$.

We describe this process of discovery in some detail here, as the general technique appears to be quite fruitful and may well yield results in other settings.

We first conjectured that $\zeta(2 n+2)$ is a rational combination of terms of the form

$$
\sigma\left(2 r ;\left[2 a_{1}, \cdots, 2 a_{N}\right]\right):=\sum_{k=1}^{\infty} \frac{1}{k^{2 r}\left(\begin{array}{c}
2 k \\
k
\end{array}\right)} \prod_{i=1}^{N} \sum_{n_{i}=1}^{k-1} \frac{1}{n_{i}^{2 a_{i}}},
$$

where $r+\sum_{i=1}^{N} a_{i}=n+1$, and the $a_{i}$ are listed in nonincreasing order (note that the right-hand-side value is independent of the order of the $a_{i}$ ). This dramatically reduces the size of the search space, while in addition the sums (37) are relatively easy to compute.

One can then write

$$
\sum_{n=0}^{\infty} \zeta(2 n+2) x^{2 n} \stackrel{?}{=} \sum_{n=0}^{\infty} \sum_{r=1}^{n+1} \sum_{\pi \in \Pi(n+1-r)} \alpha(\pi) \sigma(2 r ; 2 \pi) x^{2 n}
$$

where $\Pi(m)$ denotes the set of all additive partitions of $m$ if $m>0, \Pi(0)$ is the singleton set whose sole element is the null partition [], and the coefficients $\alpha(\pi)$ are complex numbers. In principle $\alpha(\pi)$ in (38) could depend not only on the partition $\pi$ but also on $n$. However, since the first few coefficients appeared to be independent of $n$, we found it helpful to make the ansatz that the generating function could be expressed in the form given above. 

$m$, let

For positive integer $k$ and partition $\pi=\left(a_{1}, a_{2}, \ldots, a_{N}\right)$ of the positive integer

$$
\widehat{\sigma}_{k}(\pi):=\prod_{i=1}^{N} \sum_{n_{i}=1}^{k-1} \frac{1}{n_{i}^{2 a_{i}}}
$$

Then

$$
\sigma(2 r ; 2 \pi)=\sum_{k=1}^{\infty} \frac{\widehat{\sigma}_{k}(\pi)}{k^{2 r}\left(\begin{array}{c}
2 k \\
k
\end{array}\right)}
$$

and from (38), we deduce that

$$
\begin{aligned}
\sum_{n=0}^{\infty} \zeta(2 n+2) x^{2 n} & =\sum_{n=0}^{\infty} \sum_{r=1}^{n+1} \sum_{\pi \in \Pi(n+1-r)} \alpha(\pi) \sigma(2 r ; 2 \pi) x^{2 n} \\
& =\sum_{k=1}^{\infty} \frac{1}{\left(\begin{array}{c}
2 k \\
k
\end{array}\right)} \sum_{r=1}^{\infty} \frac{x^{2 r-2}}{k^{2 r}} \sum_{n=r-1}^{\infty} \sum_{\pi \in \Pi(n+1-r)} \alpha(\pi) \widehat{\sigma}_{k}(\pi) x^{2(n+1-r)} \\
& =\sum_{k=1}^{\infty} \frac{1}{\left(\begin{array}{c}
2 k \\
k
\end{array}\right)\left(k^{2}-x^{2}\right)} \sum_{m=0}^{\infty} x^{2 m} \sum_{\pi \in \Pi(m)} \alpha(\pi) \widehat{\sigma}_{k}(\pi) \\
& =\sum_{k=1}^{\infty} \frac{1}{\left(\begin{array}{c}
2 k \\
k
\end{array}\right)\left(k^{2}-x^{2}\right)} P_{k}(x)
\end{aligned}
$$

where

$$
P_{k}(x):=\sum_{m=0}^{\infty} x^{2 m} \sum_{\pi \in \Pi(m)} \alpha(\pi) \widehat{\sigma}_{k}(\pi)
$$

whose closed form is yet to be determined. Our strategy, as in the case of (34) [22], was to compute $P_{k}(x)$ explicitly for a few small values of $k$ in a hope that these would suggest a closed form for general $k$.

Some examples we produced are shown below. At each step we "bootstrapped" by assuming that the first few coefficients of the current result are the coefficients of the previous result. Then we found the remaining coefficients (which are in each case unique) by means of PSLQ computations. Note below that in the sigma notation, the first few coefficients of each expression are simply the previous step's terms, where the first argument of $\sigma$ (corresponding to $r$ ) has been increased by two. These initial terms (with coefficients in bold) are then followed by terms with the other partitions as arguments, with all terms ordered lexicographically by partition (shorter partitions are listed before longer partitions, and, within a partition of a given length, larger entries are listed before smaller entries in the first position 
where they differ; the integers in brackets are nonincreasing):

$$
\begin{aligned}
& (40) \zeta(2)=3 \sum_{k=1}^{\infty} \frac{1}{\left(\begin{array}{c}
2 k \\
k
\end{array}\right) k^{2}}=3 \sigma(2,[0]) \\
& (41) \zeta(4)=\mathbf{3} \sum_{k=1}^{\infty} \frac{1}{\left(\begin{array}{c}
2 k \\
k
\end{array}\right) k^{4}}-9 \sum_{k=1}^{\infty} \frac{\sum_{j=1}^{k-1} j^{-2}}{\left(\begin{array}{c}
2 k \\
k
\end{array}\right) k^{2}}=\mathbf{3} \sigma(4,[0])-9 \sigma(2,[2]) \\
& \zeta(6)=\mathbf{3} \sum_{k=1}^{\infty} \frac{1}{\left(\begin{array}{c}
2 k \\
k
\end{array}\right) k^{6}}-\mathbf{9} \sum_{k=1}^{\infty} \frac{\sum_{j=1}^{k-1} j^{-2}}{\left(\begin{array}{c}
2 k \\
k
\end{array}\right) k^{4}}-\frac{45}{2} \sum_{k=1}^{\infty} \frac{\sum_{j=1}^{k-1} j^{-4}}{\left(\begin{array}{c}
2 k \\
k
\end{array}\right) k^{2}} \\
& +\frac{27}{2} \sum_{k=1}^{\infty} \sum_{j=1}^{k-1} \frac{\sum_{i=1}^{k-1} i^{-2}}{j^{2}\left(\begin{array}{c}
2 k \\
k
\end{array}\right) k^{2}} \\
& =\mathbf{3} \sigma(6,[])-\mathbf{9} \sigma(4,[2])-\frac{45}{2} \sigma(2,[4])+\frac{27}{2} \sigma(2,[2,2]) \\
& \zeta(8)=\mathbf{3} \sigma(8,[])-\mathbf{9} \sigma(6,[2])-\frac{\mathbf{4 5}}{\mathbf{2}} \sigma(4,[4])+\frac{\mathbf{2 7}}{\mathbf{2}} \sigma(4,[2,2])-63 \sigma(2,[6]) \\
& +\frac{135}{2} \sigma(2,[4,2])-\frac{27}{2} \sigma(2,[2,2,2]) \\
& \zeta(10)=\mathbf{3} \sigma(10,[])-\mathbf{9} \sigma(8,[2])-\frac{\mathbf{4 5}}{\mathbf{2}} \sigma(6,[4])+\frac{\mathbf{2 7}}{\mathbf{2}} \sigma(6,[2,2])-\mathbf{6 3} \sigma(4,[6]) \\
& +\frac{\mathbf{1 3 5}}{\mathbf{2}} \sigma(4,[4,2])-\frac{\mathbf{2 7}}{\mathbf{2}} \sigma(4,[2,2,2])-\frac{765}{4} \sigma(2,[8])+189 \sigma(2,[6,2]) \\
& +\frac{675}{8} \sigma(2,[4,4])-\frac{405}{4} \sigma(2,[4,2,2])+\frac{81}{8} \sigma(2,[2,2,2,2]) \text {. }
\end{aligned}
$$

Next from the above results, one can immediately read that $\alpha([])=3, \alpha([1])=$ $-9, \alpha([2])=-45 / 2, \alpha([1,1])=27 / 2$, and so forth. Table 3 presents the values of $\alpha$ that we obtained in this manner.

Using these values, we then calculated series approximations to the functions $P_{k}(x)$, by using formula (39). We obtained:

$$
\begin{aligned}
P_{3}(x) \approx & 3-\frac{45}{4} x^{2}-\frac{45}{16} x^{4}-\frac{45}{64} x^{6}-\frac{45}{256} x^{8}-\frac{45}{1024} x^{10}-\frac{45}{4096} x^{12}-\frac{45}{16384} x^{14} \\
& -\frac{45}{65536} x^{16} \\
P_{4}(x) \approx & 3-\frac{49}{4} x^{2}+\frac{119}{144} x^{4}+\frac{3311}{5184} x^{4}+\frac{38759}{186624} x^{6}+\frac{384671}{6718464} x^{8} \\
& +\frac{3605399}{241864704} x^{10}+\frac{33022031}{8707129344} x^{12}+\frac{299492039}{313456656384} x^{14} \\
P_{5}(x) \approx & 3-\frac{205}{16} x^{2}+\frac{7115}{2304} x^{4}+\frac{207395}{331776} x^{6}+\frac{4160315}{47775744} x^{8}+\frac{74142995}{6879707136} x^{10} \\
& +\frac{1254489515}{990677827584} x^{12}+\frac{20685646595}{142657607172096} x^{14}+\frac{336494674715}{20542695432781824} x^{16}
\end{aligned}
$$




\begin{tabular}{|l|l|l|l|l|l|}
\hline Partition & Alpha & Partition & Alpha & Partition & Alpha \\
\hline [empty] & $3 / 1$ & 1 & $-9 / 1$ & 2 & $-45 / 2$ \\
1,1 & $27 / 2$ & 3 & $-63 / 1$ & 2,1 & $135 / 2$ \\
$1,1,1$ & $-27 / 2$ & 4 & $-765 / 4$ & 3,1 & $189 / 1$ \\
2,2 & $675 / 8$ & $2,1,1$ & $-405 / 4$ & $1,1,1,1$ & $81 / 8$ \\
5 & $-3069 / 5$ & 4,1 & $2295 / 4$ & 3,2 & $945 / 2$ \\
$3,1,1$ & $-567 / 2$ & $2,2,1$ & $-2025 / 8$ & $2,1,1,1$ & $405 / 4$ \\
$1,1,1,1,1$ & $-243 / 40$ & 6 & $-4095 / 2$ & 5,1 & $9207 / 5$ \\
4,2 & $11475 / 8$ & $4,1,1$ & $-6885 / 8$ & 3,3 & $1323 / 2$ \\
$3,2,1$ & $-2835 / 2$ & $3,1,1,1$ & $567 / 2$ & $2,2,2$ & $-3375 / 16$ \\
$2,2,1,1$ & $6075 / 16$ & $2,1,1,1,1$ & $-1215 / 16$ & $1,1,1,1,1,1$ & $243 / 80$ \\
7 & $-49149 / 7$ & 6,1 & $49140 / 8$ & 5,2 & $36828 / 8$ \\
$5,1,1$ & $-27621 / 10$ & 4,3 & $32130 / 8$ & $4,2,1$ & $-34425 / 8$ \\
$4,1,1,1$ & $6885 / 8$ & $3,3,1$ & $-15876 / 8$ & $3,2,2$ & $-14175 / 8$ \\
$3,2,1,1$ & $17010 / 8$ & $3,1,1,1,1$ & $-1701 / 8$ & $2,2,2,1$ & $10125 / 16$ \\
$2,2,1,1,1$ & $-6075 / 16$ & $2,1,1,1,1,1$ & $729 / 16$ & $1,1,1,1,1,1,1$ & $-729 / 560$ \\
8 & $-1376235 / 56$ & 7,1 & $1179576 / 56$ & 6,2 & $859950 / 56$ \\
$6,1,1$ & $-515970 / 56$ & 5,3 & $902286 / 70$ & $5,2,1$ & $-773388 / 56$ \\
$5,1,1,1$ & $193347 / 70$ & 4,4 & $390150 / 64$ & $4,3,1$ & $-674730 / 56$ \\
$4,2,2$ & $-344250 / 64$ & $4,2,1,1$ & $413100 / 64$ & $4,1,1,1,1$ & $-41310 / 64$ \\
$3,3,2$ & $-277830 / 56$ & $3,3,1,1$ & $166698 / 56$ & $3,2,2,1$ & $297675 / 56$ \\
$3,2,1,1,1$ & $-119070 / 56$ & $3,1,1,1,1,1$ & $10206 / 80$ & $2,2,2,2$ & $50625 / 128$ \\
$2,2,2,1,1$ & $-60750 / 64$ & $2,2,1,1,1,1$ & $18225 / 64$ & $2,1,1,1,1,1,1$ & $-1458 / 64$ \\
$1,1,1,1,1,1,1,1$ & $2187 / 4480$ & & & & \\
\hline
\end{tabular}

TABLE 3. Alpha coefficients found by PSLQ computations

$$
\begin{aligned}
P_{6}(x) \approx & 3-\frac{5269}{400} x^{2}+\frac{6640139}{1440000} x^{4}+\frac{1635326891}{5184000000} x^{6}-\frac{5944880821}{18662400000000} x^{8} \\
& -\frac{212874252291349}{67184640000000000} x^{10}-\frac{141436384956907381}{241864704000000000000} x^{12} \\
& -\frac{70524260274859115989}{870712934400000000000000} x^{14}-\frac{31533457168819214655541}{3134566563840000000000000000} x^{16} \\
P_{7}(x) \approx & 3-\frac{5369}{400} x^{2}+\frac{8210839}{1440000} x^{4}-\frac{199644809}{5184000000} x^{6}-\frac{680040118121}{18662400000000} x^{8} \\
& -\frac{278500311775049}{67184640000000000} x^{10}-\frac{84136715217872681}{241864704000000000000} x^{12} \\
& -\frac{22363377813883431689}{870712934400000000000000} x^{14}-\frac{5560090840263911428841}{3134566563840000000000000000} x^{16} .
\end{aligned}
$$

With these unappetizing approximations in hand, we were nonetheless in a position to attempt to determine better closed-form expressions for $P_{k}(x)$. This can be done by using the "Pade" function in either Mathematica or Maple. We 
obtained the following:

$$
\begin{aligned}
& P_{1}(x) \stackrel{?}{=} 3 \\
& P_{2}(x) \stackrel{?}{=} \frac{3\left(4 x^{2}-1\right)}{\left(x^{2}-1\right)} \\
& P_{3}(x) \stackrel{?}{=} \frac{12\left(4 x^{2}-1\right)}{\left(x^{2}-4\right)} \\
& P_{4}(x) \stackrel{?}{=} \frac{12\left(4 x^{2}-1\right)\left(4 x^{2}-9\right)}{\left(x^{2}-4\right)\left(x^{2}-9\right)} \\
& P_{5}(x) \stackrel{?}{=} \frac{48\left(4 x^{2}-1\right)\left(4 x^{2}-9\right)}{\left(x^{2}-9\right)\left(x^{2}-16\right)} \\
& P_{6}(x) \stackrel{?}{=} \frac{48\left(4 x^{2}-1\right)\left(4 x^{2}-9\right)\left(4 x^{2}-25\right)}{\left(x^{2}-9\right)\left(x^{2}-16\right)\left(x^{2}-25\right)} \\
& P_{7}(x) \stackrel{?}{=} \frac{192\left(4 x^{2}-1\right)\left(4 x^{2}-9\right)\left(4 x^{2}-25\right)}{\left(x^{2}-16\right)\left(x^{2}-25\right)\left(x^{2}-36\right)} .
\end{aligned}
$$

These results immediately suggest the general form of a generating function identity:

$$
\sum_{n=0}^{\infty} \zeta(2 n+2) x^{2 n} \stackrel{?}{=} 3 \sum_{k=1}^{\infty} \frac{1}{\left(\begin{array}{c}
2 k \\
k
\end{array}\right)\left(k^{2}-x^{2}\right)} \prod_{m=1}^{k-1} \frac{4 x^{2}-m^{2}}{x^{2}-m^{2}}
$$

which is equivalent to (35).

We next confirmed this result in several ways:

(1) We symbolically computed the power series coefficients of the LHS and the RHS of (46), and verified that they agree up to the term with $x^{100}$.

(2) We verified that $\mathcal{Z}(1 / 6)$, where $\mathcal{Z}(x)$ is the RHS of $(46)$, agrees with $18-3 \sqrt{3} \pi$, computed using (36), to over 2,500 digit precision; likewise for $\mathcal{Z}(1 / 2)=2, \mathcal{Z}(1 / 3)=9 / 2-3 \pi /(2 \sqrt{3}), \mathcal{Z}(1 / 4)=8-2 \pi$ and $\mathcal{Z}(1 / \sqrt{2})=$ $1-\pi / \sqrt{2} \cdot \cot (\pi / \sqrt{2})$.

(3) We then affirmed that formula (46) gives the same numerical value as (36) for the 100 pseudorandom values $\{m \pi\}$, for $1 \leq m \leq 100$, where $\{\cdot\}$ denotes the fractional part.

Thus, we were certain that (35) was correct and it remained only to find a proof. The first component was to show, as anticipated, that (35) was equivalent to a finite identity. Indeed, in terms of the generalized hypergeometric series

$$
{ }_{3} F_{2}\left(\begin{array}{c|c}
3 n, n+1,-n \\
2 n+1, n+1 / 2 & \frac{1}{4}
\end{array}\right):=\sum_{k=0}^{n} \frac{(3 n)_{k}(n+1)_{k}(-n)_{k}}{(2 n+1)_{k}(n+1 / 2)_{k}} \cdot \frac{(1 / 4)^{k}}{k !}=\frac{\left(\begin{array}{c}
2 n \\
n
\end{array}\right)}{\left(\begin{array}{c}
3 n \\
n
\end{array}\right)}
$$

we showed (35) was equivalent to (47) holding for all integer $n>0$. Here for nonnegative integers $k$ and any $a,(a)_{k}:=\prod_{j=1}^{k}(a+j-1)=\Gamma(a+k) / \Gamma(a)$ is the usual Pochhammer symbol. This can been done (easily in this case) by applying the Wilf-Zeilberger method as implemented in Maple. See [9] for details. A human proof of (47) would be welcome, though there is no a prior reason why such a proof must exist! 


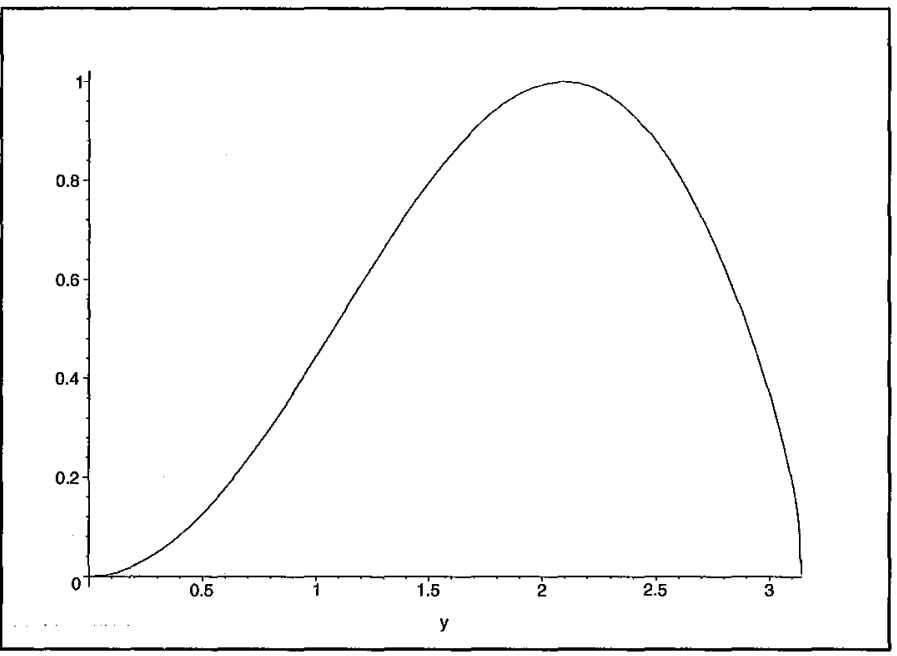

Figure 3. Plot of the Glasser-Oloa integrand with a vertical tangent at $\pi$

4.3. Another integral in search of a proof. Before concluding, we wish to mention that Glasser and Oloa recently discovered the following integral evaluation based on an exploration of integrals of Wronskians [42]:

$$
\int_{0}^{\pi} \frac{y^{2}}{y^{2}+4 \ln ^{2}\left(2 \cos \frac{y}{2}\right)} d y \stackrel{?}{=} \frac{\pi}{4}(1-\gamma)+\frac{\pi}{4} \ln (2 \pi)=1.7755193128079 \ldots
$$

At the time of the web posting, they had no rigorous proof of this identity, but we were able to quickly confirm that it held to 1,000 decimal digits, on using the tanh-sinh quadrature algorithm to evaluate the left-hand-side. Figure 3 shows the integrand in (48). Thus, while the identity here was hardly in serious doubt, we had neither a computer nor a human proof. Soon Dante Manna and Larry Glasser found elegant derivations of (48), see [28].

The 1,000 digit computation of this integral required 290 seconds (not including initialization) on a single CPU of an IBM Power3 system, a 5-year-old technology. By comparison, this same calculation required only 74 seconds on a single CPU of the more recent Apple G5, and only 30 seconds on the latest Intel-based Apple workstation. What's more, each of the two Intel processors in the new system features two computational cores, and all four of these cores can be utilized concurrently (although this was not done on this problem). Moore's law still obtains.

\section{Caveat Emptor}

Despite all of these remarkable advances, we issue a final caveat: seemingly compelling patterns can mislead, and thus caution must be exercised in all conclusions based on such patterns. 
As a single instance, it was discovered by Robert Baillie [2], after reading [20, $\S 2.5]$, that for $N=1,2,3,4,5$, and 6 (but not 7 ),

$$
\sum_{n=1}^{\infty} \operatorname{sinc}^{N}(n)=-\frac{1}{2}+\int_{0}^{\infty} \operatorname{sinc}^{N}(x) d x .
$$

Numerous other examples could be cited-see for instance [5, pg. 511-512]. These are cautionary examples of that perennial issue of inductive mathematics: When is enough evidence enough? One example in [2] holds for more than $10^{176}$ cases and fails thereafter by less than one part in a googolplex.

\section{Conclusion}

With the increasing numbers of computational papers being published in the field, it is clear that "experimental" or "computer-assisted" mathematics is truly emerging as an important new paradigm in mathematical research. One aspect of this discipline that is particularly encouraging is its democratic nature.

For one thing, because the objects typically studied in this arena are typically rather basic and concrete, researchers of a wide range of backgrounds can participate. Recent contributions of note have been made by undergraduates, senior researchers, and also computer scientists and mathematical physicists. The only essential prerequisite is a reasonably good facility with computation of one sort or another.

What's more, the results of these computations are also democratizing, because with the numerical results in hand, researchers who are not specialists in the computational techniques involved can utilize these results in research. This was driven home to the present authors recently when one of us sent to Richard Crandall a numerical value we obtained for the constant $P(3)$ mentioned in Section 3.4 above. He in turn emailed the value to Aaron Goldberg, a graduate research assistant, who by means of an Internet search found a mathematical physics paper ([16]) that discussed this constant. We already knew about this paper, but Goldberg found it by himself without guidance.

The democratizing nature of computational mathematics was also noted in press reports of the recent computation of the representations for the symmetry group E8 [36]. This computation, by the way, produced a square matrix of coefficients 453,060 on a side, with $205,263,363,600$ entries. It is part of a project started by Jeffrey Adams of the University of Maryland to create an "Atlas of Lie Groups and Representations," as an aid researchers in the field. Adams noted that the computation is like the "genome" for E8. "It's all of the information you need to understand $\mathrm{E} 8$ and its representations."

So where will all this lead? As we emphasized in the introduction, numerous useful and impressive mathematical software packages are now available (for free!) on the Internet. And this is in addition to commercial packages, such as Mathematica and Maple, which grow more powerful and reliable with each new release. Further, Moore's Law shows no sign of abating. If anything, some of the astonishing new developments in the field of nanotechnology (see for instance [35]) suggest that the current rapid pace of advancement might even accelerate somewhat in the coming years.

So imagine a future when high-dimensional integrals can routinely be evaluated to hundreds or thousands of digits of precision. Perhaps then we could make rapid 
progress on the Boos-Korepin conjecture of Section 3.4. Or imagine a future when hundreds or thousands of candidate terms can routinely be incorporated into an integer relation calculation, thus permitting a much broader search of possible terms for analytic evaluation. Or imagine the time when an enormous computation of scope comparable to the above-mentioned E8 calculation, which today required three days of processing on a large computer cluster (not to mention the many hours of programming and preparation), could be dispatched in an hour or two on an individual researcher's desktop system. Even more importantly, imagine a future when many thousands of mathematicians are completely fluent in using these (and even more powerful, yet-to-be-developed) computer-based tools in their research work.

That future is coming! Indeed, as the novelist William Gibson-who named cyber-space years before he owned a computer-declared in 1999, "The future is already here. It's just not very evenly distributed."

\section{References}

[1] M. Ambramowitz and I. A. Stegun, Handbook of Mathematical Functions, NBS (now NIST), 1965.

[2] Robert Baillie, David Borwein and Jonathan Borwein, "Some sinc sums and integrals," MAA Monthly, in press 2008. [D-drive Preprint 332].

[3] David H. Bailey, "Integer Relation Detection," Computing in Science and Engineering, Jan-Feb, 2000, pg. 24-28.

[4] David H. Bailey, "A Compendium of BBP-Type Formulas for Mathematical Constants," manuscript, 2000, available at http://crd.lbl.gov/ “dhbailey/dhbpapers/bbp-formulas.pdf.

[5] David H. Bailey and Jonathan M. Borwein, "Experimental Mathematics: Examples, Methods and Implications," Notices of the American Mathematical Society, vol. 52, no. 5 (May 2005), pg. 502-514, available at http://www . ams .org/notices/200505/fea-borwein. pdf.

[6] D. Bailey, J. Borwein, N. Calkin, R. Girgensohn, R. Luke, and V. Moll, Experimental Mathematics in Action, AK Peters, 2007. ISBN: 978-1-56881-271-7.

[7] David H. Bailey, David Borwein, Jonathan M. Borwein and Richard Crandall, "Hypergeometric Forms for Ising-Class Integrals," Experimental Mathematics, to appear, Jul 2006, available at http://crd.1bl.gov/ dhbailey/dhbpapers/meijer.pdf.

[8] David H. Bailey and Jonathan M. Borwein, "Highly Parallel, High-Precision Numerical Integration," manuscript, 2006, available at http://crd.lbl.gov/ ${ }^{\sim}$ dhbailey/dhbpapers/quadparallel.pdf.

[9] David H. Bailey, Jonathan M. Borwein and David M. Bradley, "Experimental Determination of Apery-Like Identities for Zeta $(2 n+2)$," Experimental Mathematics, vol. 15 (2006), pg. 281-289.

[10] David H. Bailey, Jonathan M. Borwein and Richard E. Crandall, "Integrals of the Ising Class," Journal of Physics A: Mathematical and General, to appear, Jun 2006; available at http://crd.lbl.gov/ dhbailey/dhbpapers/ising.pdf.

[11] David H. Bailey and David J. Broadhurst, "Parallel Integer Relation Detection: Techniques and Applications," Mathematics of Computation, vol. 70, no. 236 (Oct 2000), pg. 1719-1736.

[12] David H. Bailey, Xiaoye S. Li and Karthik Jeyabalan, "A Comparison of Three High-Precision Quadrature Schemes," Experimental Mathematics, vol. 14 (2005), no. 3, pg 317-329.

[13] W. N. Bailey, "Generalized Hypergeometric Series," Cambridge University Press, Cambridge, 1935.

[14] Michael S. Becker, "Problem 11275," American Mathematical Monthly, vol. 11, no. 2 (Feb. 2007), pg 165.

[15] H. E. Boos and V. E. Korepin, "Quantum Spin Chains and Riemann Zeta Function with Odd Arguments," Journal of Physics A: Mathematics and General, vol. 34 (2001), pg. 5311-5316; hep-th/0104008. 
[16] H. E. Boos, V. E. Korepin, Y. Nishiyama and M. Shiroishi, "Quantum Correlations and Number Theory," Journal of Physics A: Mathematics and General, vol. 35 (2002), pg. 4443, availabe at

http://www . citebase .org/abstract?id=oai : arXiv . org: cond-mat/0202346.

[17] Folkmar Bornemann and Dirk Laurie, Stan Wagon and Jorg Waldvogel, The SIAM 100-Digit Challenge, SIAM, Philadelphia, 2004.

[18] J.M. Borwein, "The SIAM 100 Digits Challenge," Extended review for the Mathematical Intelligencer, vol. 27, no. 4 (2005), pg 40-48.

[19] Jonathan M. Borwein and David H. Bailey, Mathematics by Experiment: Plausible Reasoning in the 21st Century, AK Peters, Natick, MA, 2004.

[20] Jonathan M. Borwein, David H. Bailey and Roland Girgensohn, Experimentation in Mathematics: Computational Paths to Discovery, AK Peters, Natick, MA, 2004.

[21] Jonathan M. Borwein and David M. Bradley, "Empirically Determined Apéry-like Formulae for $\zeta(4 n+3)$," Experimental Mathematics, vol. 6 (1997), pg. 181-194.

[22] Jonathan M. Borwein and David M. Bradley, "Searching Symbolically for Apéry-like Formulae for Values of the Riemann Zeta Function," ACM SIGSAM Bulletin of Algebraic and Symbolic Manipulation, vol. 30 (1996), pg. 2-7.

[23] Jonathan Borwein and David Broadhurst, "Determination of Rational Dirichlet-zeta Invariants of Hyperbolic Manifolds and Feymman Knots and Links," 1998, available at http: //arxiv . org/hep-th/9811173.

[24] Jonathan M. Borwein, David J. Broadhurst and Joel Kamnitzer, "Central Binomial Sums, Multiple Clausen Values, and Zeta Values," Experimental Mathematics, vol. 10 (2001), pg. 25-41.

[25] Jonathan M. Borwein and Peter B. Borwein, Pi and the AGM: A Study in Analytic Number Theory and Computational Complexity, CMS Series of Mongraphs and Advanced books in Mathematics, John Wiley, Hoboken, NJ, 1987.

[26] Jonathan Borwein and Bruno Salvy, A proof of a recursion for Bessel moments, D-drive Preprint 346, 2007.

[27] Louis Comtet, Advanced Combinatorics: The Art of Finite and Infinite Expansion, D. Reidel Publishing, Dordrecht, Holland, 1974.

[28] Larry Glasser and Dante Manna, "On the Lapalace transform of the psi-function," Contemporary Mathematics, to appear, 2008.

[29] Kate Greene, "The Promise of Personal Supercomputers," Technology Review, 23 Feb 2007, available at http://www.technologyreview.com/Infotech/18219.

[30] Jesus Guillera, "Some Binomial Series Obtained by the WZ-Method," Advances in Applied Mathematics, vol. 29 (2002), pg. 599-603.

[31] Jesus Guillera, "Generators of Some Ramanujan Formulas,", Ramanujan Journal, vol. 11 (2006), pg. 41-48.

[32] Jesus Guillera, "About a New Kind of Ramanujan-Type Series," Experimental Mathematics, vol. 12 (2003), pg. 507-510.

[33] F. Gygi, E. W. Draeger, M. Schulz, B. R. de Supinski, J. A. Gunnels, V. Austel, J. C. Sexton, F. Franchetti, S. Kral, C. W. Ueberhuber and J. Lorenz, "Large-Scale Electronic Structure Calculations of High-Z Metals on the BlueGene/L Platform," Proceedings of SC2006, Nov. 2006, available at http://sc06. supercomputing. org/schedule/pdf/gb104.pdf.

[34] Max Koecher, "Letter (German)," Mathematics Intelligencer, vol. 2 (1979/80), pg. 62-64.

[35] John Markoff, "H.P. to Report an Advance in Adaptable Circuitry," New York Times, 16 Jan 2007, available at http://www .nytimes . com/2007/01/16/technology/16nano.html.

[36] J. R. Minkel, "Mathematicians Team Up with Supercomputer to Crack 248-Dimensional Object," 19 Mar 2007, available at http://www . sciam.com/article.cfm?articleid=6C66165B-E7F2-99DF3D86B476FFD18F17.

[37] Gordon E. Moore, "Cramming More Components onto Integrated Circuits," Electronics Magazine, 19 April 1965, available at http://download.intel . com/research/silicon/moorespaper .pdf.

[38] Marko Petkovsek, Herbert S. Wilf and Doron Zeilberger, $A=B$, AK Peters, Natick, MA, 1996. 
[39] Richard Preston, "The Mountains of Pi," New Yorker, Mar. 2, 1992, available at http://www. newyorker.com/archive/content/articles/050411fr_archive01.

[40] Katherine Unger, "Helaman Ferguson Profile: Carving His Own Unique Niche, in Symbols and Stone," Science, vol. 314 (2006), no. 5798, 20 Oct 2006, pg 412-413.

[41] Alfred van der Poorten, "A Proof that Euler Missed: Apéry's Proof of the Irrationality of $\zeta(3)^{\prime \prime}$, Mathematical Intelligencer, vol. 1 (1978/79), pg. 195-203.

[42] Eric W. Weisstein, "Definite Integral," MathWorld-A Wolfram Web Resource, http://mathworld.wolfram.com/DefiniteIntegral.html.

[43] Doron Zeilberger, "Identities in Search of Identity," Theoretical Computer Science, vol. 117 (1993), pg. 23-38.

LaWrence Berkeley National Laboratory, Berkeley, CA 94720

E-mail address: dhbailey@lbl.gov

Faculty of Computer Science, Dalhousie University, Halifax, NS, B3H 2W5, Canada

E-mail address: jborwein@cs.dal.ca 